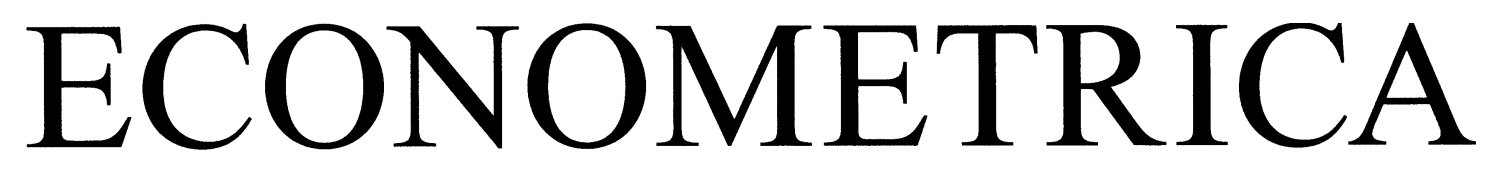

JOURNAL OF THE ECONOMETRIC SOCIETY

An International Society for the Advancement of Economic Theory in its Relation to Statistics and Mathematics

http://www.econometricsociety.org/

Econometrica, Vol. 83, No. 4 (July, 2015), 1543-1579

\title{
LINEAR REGRESSION FOR PANEL WITH UNKNOWN NUMBER OF FACTORS AS INTERACTIVE FIXED EFFECTS
}

\author{
HyungsiK Roger MoON \\ USC Dornsife INET, University of Southern California, Los Angeles, CA \\ 90089-0253, U.S.A. and Yonsei University, Seoul, Korea \\ MARTIN WEIDNER \\ University College London, London, WC1E 6BT, U.K. and CeMMaP
}

The copyright to this Article is held by the Econometric Society. It may be downloaded, printed and reproduced only for educational or research purposes, including use in course packs. No downloading or copying may be done for any commercial purpose without the explicit permission of the Econometric Society. For such commercial purposes contact the Office of the Econometric Society (contact information may be found at the website http://www.econometricsociety.org or in the back cover of Econometrica). This statement must be included on all copies of this Article that are made available electronically or in any other format. 


\title{
NOTES AND COMMENTS
}

\section{LINEAR REGRESSION FOR PANEL WITH UNKNOWN NUMBER OF FACTORS AS INTERACTIVE FIXED EFFECTS}

\author{
By HyungsiK Roger MoOn AND MARTIN WEIDNER ${ }^{1}$
}

\begin{abstract}
In this paper, we study the least squares (LS) estimator in a linear panel regression model with unknown number of factors appearing as interactive fixed effects. Assuming that the number of factors used in estimation is larger than the true number of factors in the data, we establish the limiting distribution of the LS estimator for the regression coefficients as the number of time periods and the number of cross-sectional units jointly go to infinity. The main result of the paper is that under certain assumptions, the limiting distribution of the LS estimator is independent of the number of factors used in the estimation as long as this number is not underestimated. The important practical implication of this result is that for inference on the regression coefficients, one does not necessarily need to estimate the number of interactive fixed effects consistently.
\end{abstract}

KEYWORDS: Panel data, interactive fixed effects, factor models, perturbation theory of linear operators, random matrix theory.

\section{INTRODUCTION}

PANEL DATA MODELS TYPICALLY INCORPORATE INDIVIDUAL AND TIME EFFECTS to control for heterogeneity in cross section and over time. While often these individual and time effects enter the model additively, they can also be interacted multiplicatively, thus giving rise to so-called interactive effects, which we also refer to as a factor structure. The multiplicative form captures the heterogeneity in the data more flexibly, since it allows for common timevarying shocks (factors) to affect the cross-sectional units with individual specific sensitivities (factor loadings). ${ }^{2}$ It is this flexibility that motivated the discussion of interactive effects in the econometrics literature, for example, HoltzEakin, Newey, and Rosen (1988), Ahn, Lee, and Schmidt (2001, 2013), Pesaran (2006), Bai (2009a, 2013), Zaffaroni (2009), Moon and Weidner (2014), and Lu and $\mathrm{Su}$ (2013).

\footnotetext{
${ }^{1}$ We thank the participants of the 2009 Cowles Summer Conference "Handling Dependence: Temporal, Cross-Sectional, and Spatial" at Yale University, the 2012 North American Summer Meeting of the Econometric Society at Northwestern University, the 18th International Conference on Panel Data at the Banque de France, the 2013 North American Winter Meeting of the Econometric Society in San Diego, the 2014 Asia Meeting of Econometric Society in Taipei, the 2014 Econometric Study Group Conference in Bristol, and the econometrics seminars at USC and Toulouse for many interesting comments, and we thank Dukpa Kim, Tatsushi Oka, and Alexei Onatski for helpful discussions. We are also grateful for the comments and suggestions of the journal editors and anonymous referees. Moon acknowledges financial support from the NSF via Grant SES-0920903 and the faculty grant award from USC. Weidner acknowledges support from the Economic and Social Research Council through ESRC Centre for Microdata Methods and Practice Grant RES-589-28-0001.

${ }^{2}$ The conventional additive model can be interpreted as a two factor interactive fixed effects model.
} 
Let $N$ be the number of cross-sectional units, $T$ be the number of time periods, $K$ be the number of regressors, and $R^{0}$ be the true number of interactive fixed effects. We consider a linear regression model with observed outcomes $Y$, regressors $X_{k}$, and unobserved error structure $\varepsilon$, namely

$$
Y=\sum_{k=1}^{K} \beta_{k}^{0} X_{k}+\varepsilon, \quad \varepsilon=\lambda^{0} f^{0 \prime}+e,
$$

where $Y, X_{k}, \varepsilon$, and $e$ are $N \times T$ matrices, $\lambda^{0}$ is an $N \times R^{0}$ matrix, $f^{0}$ is a $T \times R^{0}$ matrix, and the regression parameters $\beta_{k}^{0}$ are scalars-the superscript zero indicates the true value of the parameters. We write $\beta$ for the $K$-vector of regression parameters, and we denote the components of the different matrices by $Y_{i t}, X_{k, i t}, e_{i t}, \lambda_{i r}^{0}$, and $f_{t r}^{0}$, where $i=1, \ldots, N, t=1, \ldots, T$, and $r=1, \ldots, R^{0}$. It is convenient to introduce the notation $\beta \cdot X:=\sum_{k=1}^{K} \beta_{k} X_{k}$. All matrices, vectors, and scalars in this paper are real valued.

We consider the interactive fixed effect specification, that is, we treat $\lambda^{0}$ and $f^{0}$ as nuisance parameters, which are estimated jointly with the parameters of interest $\beta .{ }^{3}$ The advantages of the fixed effects approach are, for instance, that it is semiparametric, since no assumption on the distribution of the interactive effects needs to be made, and that the regressors can be arbitrarily correlated with the interactive effect parameters.

We study the least squares (LS) estimator of model (1.1), which minimizes the sum of squared residuals to estimate the unknown parameters $\beta, \lambda$, and $f .{ }^{4}$ To our knowledge, this estimator was first discussed in Kiefer (1980). Under an asymptotic where $N$ and $T$ grow to infinity, the asymptotic properties of the LS estimator were derived in Bai (2009a) for strictly exogeneous regressors, and were extended in Moon and Weidner (2014) to the case of predetermined regressors.

An important restriction of these papers is that the number of factors $R^{0}$ is assumed to be known. However, in many empirical applications, there is no consensus about the exact number of factors in the data or in the relevant economic model. If $R^{0}$ is not known beforehand, then it may be estimated consistently, ${ }^{5}$ but difficulties in obtaining reliable estimates for the number of

\footnotetext{
${ }^{3}$ When we refer to interactive fixed effects, we mean that both factors and factor loadings are treated as nonrandom parameters. Ahn, Lee, and Schmidt (2001) take a hybrid approach in that they treat the factors as nonrandom, but treat the factor loadings as random. The common correlated effects estimator of Pesaran (2006) was introduced in a context where both the factor loadings and the factors follow certain probability laws, but it exhibits many properties of a fixed effects estimator.

${ }^{4}$ The LS estimator is sometimes called concentrated least squares estimator in the literature, and in an earlier version of the paper, we referred to it as the "Gaussian quasi maximum likelihood estimator," since LS estimation is equivalent to maximizing a conditional Gaussian likelihood function. Note also that for fixed $\beta$, the LS estimator for $\lambda$ and $f$ is simply the principal components estimator.

${ }^{5}$ See the discussion in the Supplemental Material of Bai (2009b) regarding estimation of $R^{0}$.
} 
factors are well documented in the literature (see, e.g., the simulation results in Onatski (2010) and also our empirical illustration in Section 5). Furthermore, so as to use the existing inference results on $R^{0}$, one still needs a good preliminary estimator for $\beta$, so that working out the asymptotic properties of the LS estimator for $R \geq R^{0}$ is still useful when taking that route.

We investigate the asymptotic properties of the LS estimator when the true number of factors $R^{0}$ is unknown and $R\left(\geq R^{0}\right)$ number of factors are used in the estimation. ${ }^{6}$ We denote this estimator by $\widehat{\beta}_{R}$.

The main result of the paper, presented in Section 3, is that under certain assumptions, the LS estimator $\widehat{\beta}_{R}$ has the same limiting distribution as $\widehat{\beta}_{R^{0}}$ for any $R \geq R^{0}$ under an asymptotic where both $N$ and $T$ become large, while $R^{0}$ and $R$ are constant. This implies that the LS estimator $\widehat{\beta}_{R}$ is asymptotically robust toward inclusion of extra interactive effects in the model, and within the LS estimation framework, there is no asymptotic efficiency loss from choosing $R$ larger than $R^{0}$. The important empirical implication of our result is that the number of factors $R^{0}$ need not be known or estimated accurately to apply the LS estimator.

To derive this robustness result, we impose more restrictive conditions than those typically assumed with known $R^{0}$. These include that the errors $e_{i t}$ are independent and identically (i.i.d.) normally distributed and that the regressors are composed of a "low-rank" strictly stationary component, a "high-rank" strictly stationary component, and a "high-rank" predetermined component. ${ }^{7}$ Notice that while some of these restrictions are necessary for our robustness result, some of them (e.g., i.i.d. normality of $e_{i t}$ ) are imposed for technical reasons, because in the proof we use certain results from the theory of random matrices that are currently only available in that case (see the discussion in Section 4.3). To demonstrate robustness of the result, in the Monte Carlo simulations in Section 6, we consider data generating processes (DGPs) that violate some technical conditions.

Under less restrictive assumptions, we provide intermediate results that sequentially lead to the main result in Section 4 and Appendixes A.3 and A.4. In Section 4.1, we show $\sqrt{\min (N, T)}$ consistency of the LS estimator $\widehat{\beta}_{R}$ as $N, T \rightarrow \infty$ under very mild regularity conditions on $X_{i t}$ and $e_{i t}$, and without imposing any assumptions on $\lambda^{0}$ and $f^{0}$ apart from $R \geq R^{0}$. We thus obtain consistency of the LS estimator not only for an unknown number of factors, but also for weak factors, ${ }^{8}$ which is an important robustness result.

\footnotetext{
${ }^{6}$ For $R<R^{0}$, the LS estimator can be inconsistent, since then there are interactive fixed effects in the model that can be correlated with the regressors but are not controlled for in the estimation. We therefore restrict attention to the case $R \geq R^{0}$.

${ }^{7}$ The predetermined component of the regressors allows for linear feedback of $e_{i t}$ into future realizations of $X_{k, i t}$.

${ }^{8}$ See Onatski $(2010,2012)$ and Chudik, Pesaran, and Tosetti (2011) for a discussion of "strong" versus "weak" factors in factor models.
} 
In Section 4.2 we derive an asymptotic expansion of the LS profile objective function that concentrates out $f$ and $\lambda$ for the case $R=R^{0}$. Given that the profile objective function is a sum of eigenvalues of a covariance matrix, its quadratic approximation is challenging because the derivatives of the eigenvalues with respect to $\beta$ are not generally known. We thus cannot use a conventional Taylor expansion, but instead apply the perturbation theory of linear operators to derive the approximation.

In Section 4.3, we provide an example that satisfies the typical assumptions imposed with known $R^{0}$, so that $\widehat{\beta}_{R^{0}}$ is $\sqrt{N T}$ consistent, but we show that $\widehat{\beta}_{R}$ with $R>R^{0}$ is only $\sqrt{\min (N, T)}$ consistent in that example. This shows that stronger conditions are required to derive our main result.

In Appendix A.3, we show faster than $\sqrt{\min (N, T)}$ convergence of $\widehat{\beta}_{R}$ under assumptions that are less restrictive than those employed for the main result, in particular allowing for either cross-sectional or time-serial correlation of the errors $e_{i t}$. In Appendix A.4, we provide an alternative version of our main result of asymptotic equivalence of $\widehat{\beta}_{R^{0}}$ and $\widehat{\beta}_{R}, R \geq R^{0}$, which is derived under high-level assumptions.

In Section 5, we follow Kim and Oka (2014) in employing the interactive fixed effects specification to study the effect of U.S. divorce law reforms on divorce rates. This empirical example illustrates that the estimates for the coefficient $\beta$ indeed become insensitive to the choice of $R$, once $R$ is chosen sufficiently large, as expected from our theoretical results.

Section 6 contains Monte Carlo simulation results for a static panel model. For the simulations, we consider a DGP that violates the i.i.d. normality restriction of the error term. The simulation results confirm our main result of the paper even with a relatively small sample size (e.g., $N=100, T=10$ ) and non-i.i.d.-normal errors. In the Supplemental Material (Moon and Weidner (2015)), we report the Monte Carlo simulation results of an AR(1) panel model. It also confirms the robustness result in large samples, but in finite samples it shows more inefficiency than the static case. In general, one should expect some finite sample inefficiency from overestimating the number of factors when the sample size is small or the number of overfitted factors is large.

A few words on notation. The transpose of a matrix $A$ is denoted by $A^{\prime}$. For a column vector $v$, its Euclidean norm is defined by $\|v\|=\sqrt{v^{\prime} v}$. For an $m \times n$ matrix $A$, the Frobenius or Hilbert Schmidt norm is $\|A\|_{\mathrm{HS}}=\sqrt{\operatorname{Tr}\left(A A^{\prime}\right)}$ and the operator or spectral norm is $\|A\|=\max _{0 \neq v \in \mathbb{R}^{n}} \frac{\|A v\|}{\|v\|}$. Furthermore, we use $P_{A}=A\left(A^{\prime} A\right)^{\dagger} A^{\prime}$ and $M_{A}=\mathbb{1}-A\left(A^{\prime} A\right)^{\dagger} A^{\prime}$, where $\mathbb{1}$ is the $m \times m$ identity matrix and $\left(A^{\prime} A\right)^{\dagger}$ denotes some generalized inverse in case $A$ is not of full column rank. For square matrices $B$ and $C$, we use $B>C$ (or $B \geq C$ ) to indicate that $B-C$ is positive (semi) definite. We use w.p.a.1 to denote with probability approaching 1 . 


\section{IDENTIFICATION OF $\beta^{0}, \lambda^{0} f^{0 \prime}$, AND $R^{0}$}

In this section, we provide a set of conditions under which the regression coefficient $\beta^{0}$, the interactive fixed effects $\lambda^{0} f^{0 \prime}$, and the number of factors $R^{0}$ are determined uniquely by the data. Here, and throughout the whole paper, we treat $\lambda$ and $f$ as nonrandom parameters, that is, all stochastics in the following discussion are implicitly conditional on $\lambda$ and $f$. Let $x_{k}=\operatorname{vec}\left(X_{k}\right)$, the $N T$ vectorization of $X_{k}$, and let $x=\left(x_{1}, \ldots, x_{K}\right)$, which is an $N T \times K$ matrix.

ASSUMPTION ID-Assumptions for Identification: There exists a nonnegative integer $R$ such that the following statements hold:

(i) The second moments of $X_{i t}$ and $e_{i t}$ exist for all $i, t$.

(ii) We have $\mathbb{E}\left(e_{i t}\right)=0$ and $\mathbb{E}\left(X_{i t} e_{i t}\right)=0$ for all $i$, $t$.

(iii) We have $\mathbb{E}\left[x^{\prime}\left(M_{F} \otimes M_{\lambda^{0}}\right) x\right]>0$, for all $F \in \mathbb{R}^{T \times R}$.

(iv) We have $R \geq R^{0}:=\operatorname{rank}\left(\lambda^{0} f^{0 \prime}\right)$.

THEOREM 2.1-Identification: Suppose that the Assumptions ID are satisfied. Then $\beta^{0}, \lambda^{0} f^{0 \prime}$, and $R^{0}$ are identified. ${ }^{9}$

Assumption ID(i) imposes the existence of second moments. Assumption ID(ii) is an exogeneity condition, which demands that $x_{i t}$ and $e_{i t}$ are not correlated contemporaneously, but allows for predetermined regressors like lagged dependent variables. Assumption ID(iv) imposes that the true number of factors $R^{0}:=\operatorname{rank}\left(\lambda^{0} f^{0 \prime}\right)$ is bounded by a nonnegative integer $R$, which cannot be too large (e.g., the trivial bound $R=\min (N, T)$ is not possible), since otherwise Assumption ID(iii) cannot be satisfied.

Assumption ID(iii) is a noncollinearity condition, which demands that the regressors have significant variation across $i$ and over $t$ after projecting out all variation that can be explained by the factor loadings $\lambda^{0}$ and by arbitrary factors $F \in \mathbb{R}^{T \times R}$. This generalizes the within variation assumption in the conventional panel regression with time-invariant individual fixed effects, which in our notation reads $\mathbb{E}\left[x^{\prime}\left(M_{1_{T}} \otimes \mathbb{1}_{N}\right) x\right]>0 .{ }^{10}$ This conventional fixed effect assumption rules out time-invariant regressors. Similarly, Assumption ID(iii) rules out more general low-rank regressors, ${ }^{11}$ see our discussion of Assumption $\mathrm{NC}$ below.

\footnotetext{
${ }^{9}$ Here, identification means that $\beta^{0}$ and $\lambda^{0} f^{0 \prime}$ can be uniquely recovered from the distribution of $(Y, X)$ conditional on those parameters. Identification of the number of factors follows since $R^{0}=\operatorname{rank}\left(\lambda^{0} f^{0 \prime}\right)$. The factor loadings and factors $\lambda^{0}$ and $f^{0}$ are not separately identified without further normalization restrictions, but the product $\lambda^{0} f^{0 \prime}$ is identified.

${ }^{10}$ The conventional panel regression with additive individual fixed effects and time effects requires a noncollinearity condition of the form $\mathbb{E}\left[x^{\prime}\left(M_{1_{T}} \otimes M_{1_{N}}\right) x\right]>0$.

${ }^{11}$ We do not consider such low-rank regressors in this paper. Note also that Assumption A in Bai (2009a) is the sample version of our Assumption ID(iii).
} 


\section{MAIN RESULT}

The estimator we investigate in this paper is the least squares (LS) estimator, which for a given choice of $R$ reads ${ }^{12}$

$$
\left(\widehat{\beta}_{R}, \widehat{\Lambda}_{R}, \widehat{F}_{R}\right) \in \underset{\left\{\beta \in \mathbb{R}^{K}, \Lambda \in \mathbb{R}^{N \times R}, F \in \mathbb{R}^{T \times R}\right\}}{\operatorname{argmin}}\left\|Y-\beta \cdot X-\Lambda F^{\prime}\right\|_{\mathrm{HS}}^{2},
$$

where $\|\cdot\|_{\text {HS }}$ refers to the Hilbert Schmidt norm, also called the Frobenius norm. The objective function $\left\|Y-\beta \cdot X-\Lambda F^{\prime}\right\|_{\mathrm{HS}}^{2}$ is simply the sum of squared residuals. The estimator for $\beta^{0}$ can equivalently be defined by minimizing the profile objective function that concentrates out the $R$ factors and the $R$ factor loadings, namely

$$
\widehat{\beta}_{R}=\underset{\beta \in \mathbb{R}^{K}}{\operatorname{argmin}} \mathcal{L}_{N T}^{R}(\beta),
$$

with $^{13}$

$$
\begin{aligned}
\mathcal{L}_{N T}^{R}(\beta) & =\min _{\left\{\Lambda \in \mathbb{R}^{N \times R}, F \in \mathbb{R}^{T \times R}\right\}} \frac{1}{N T}\left\|Y-\beta \cdot X-\Lambda F^{\prime}\right\|_{\mathrm{HS}}^{2} \\
& =\min _{F \in \mathbb{R}^{T \times R}} \frac{1}{N T} \operatorname{Tr}\left[(Y-\beta \cdot X) M_{F}(Y-\beta \cdot X)^{\prime}\right] \\
& =\frac{1}{N T} \sum_{r=R+1}^{T} \mu_{r}\left[(Y-\beta \cdot X)^{\prime}(Y-\beta \cdot X)\right],
\end{aligned}
$$

where $\mu_{r}(\cdot)$ is the $r$ th largest eigenvalue of the matrix argument. Here, we first concentrated out $\Lambda$ by use of its own first order condition. The resulting optimization problem for $F$ is a principal components problem, so that the optimal $F$ is given by the $R$ largest principal components of the $T \times T$ matrix $(Y-\beta \cdot X)^{\prime}(Y-\beta \cdot X)$. At the optimum, the projector $M_{F}$ therefore exactly projects out the $R$ largest eigenvalues of this matrix, which gives rise to the final formulation of the profile objective function as the sum over its $T-R$ smallest eigenvalues. ${ }^{14}$ We write $\mathcal{L}_{N T}^{0}(\beta)$ for $\mathcal{L}_{N T}^{R^{0}}(\beta)$, the profile objective function obtained for the true number of factors. Notice that in (3.2), the parameter set

${ }^{12}$ The optimal $\widehat{\Lambda}_{R}$ and $\widehat{F}_{R}$ in (3.1) are not unique, since the objective function is invariant under right multiplication of $\Lambda$ with a nondegenerate $R \times R$ matrix $S$ and simultaneous right multiplication of $F$ with $\left(S^{-1}\right)^{\prime}$. However, the column spaces of $\widehat{\Lambda}_{R}$ and $\widehat{F}_{R}$ are uniquely determined.

${ }^{13}$ The profile objective function $\mathcal{L}_{N T}^{R}(\beta)$ need not be convex in $\beta$ and can have multiple local minima. Depending on the dimension of $\beta$, one should either perform an initial grid search or try multiple starting values for the optimization when calculating the global minimum $\widehat{\beta}_{R}$ numerically. See also Section S.8 of the Supplemental Material.

${ }^{14}$ This last formulation of $\mathcal{L}_{N T}^{R}(\beta)$ is very convenient since it does not involve any explicit optimization over nuisance parameters. Numerical calculation of eigenvalues is very fast, so that 
for $\beta$ is the whole Euclidean space $\mathbb{R}^{K}$ and we do not restrict the parameter set to be compact.

ASSUMPTION SF-Strong Factor Assumption:

(i) We have $0<\operatorname{plim}_{N, T \rightarrow \infty} \frac{1}{N} \lambda^{0 \prime} \lambda^{0}<\infty$.

(ii) We have $0<\operatorname{plim}_{N, T \rightarrow \infty} \frac{1}{T} f^{0 \prime} f^{0}<\infty$.

ASSUMPTION NC-Noncollinearity of $X_{k}$ : Consider linear combinations $\alpha \cdot X:=\sum_{k=1}^{K} \alpha_{k} X_{k}$ of the regressors $X_{k}$ with $K$-vector $\alpha$ such that $\|\alpha\|=1$. We assume that there exists a constant $b>0$ such that

$$
\min _{\left\{\alpha \in \mathbb{R}^{K},\|\alpha\|=1\right\}} \sum_{r=R+R^{0}+1}^{T} \mu_{r}\left[\frac{(\alpha \cdot X)^{\prime}(\alpha \cdot X)}{N T}\right] \geq b \quad \text { w.p.a.1. }
$$

AssuMPTION LL-Low Level Conditions for the Main Result:

(i) Decomposition of Regressors: We have $X_{k}=\bar{X}_{k}+\widetilde{X}_{k}^{\text {str }}+\tilde{X}_{k}^{\text {weak }}$ for $k=1, \ldots, K$, where $\bar{X}_{k}, \tilde{X}_{k}^{\text {str }}$, and $\tilde{X}_{k}^{\text {weak }}$ are $N \times T$ matrices, and the following statements hold:

(a) Low-Rank (strictly exogenous) Part of Regressors: We have that $\operatorname{rank}\left(\bar{X}_{k}\right)$ is bounded as $N, T \rightarrow \infty$ and $\frac{1}{N T} \sum_{i=1}^{N} \sum_{t=1}^{T} \bar{X}_{k, i t}^{2}=\mathcal{O}_{P}(1)$.

(b) High-Rank (strictly exogenous) Part of Regressors: We have $\left\|\widetilde{X}_{k}^{\text {str }}\right\|=$ $\mathcal{O}_{P}\left(N^{3 / 4}\right)$, as can be justified, for example, by Lemma A.1 in the Appendix.

(c) Weakly Exogenous Part of Regressors: We have $\tilde{X}_{k, i t}^{\mathrm{weak}}=\sum_{\tau=1}^{t-1} \gamma_{\tau} e_{i, t-\tau}$, where the real valued coefficients $\gamma_{\tau}$ satisfy $\sum_{\tau=1}^{\infty}\left|\gamma_{\tau}\right|<\infty$.

(d) Bounded Moments: We assume that $\mathbb{E}\left|X_{k, i t}\right|^{2}, \mathbb{E}\left|\left(M_{\lambda^{0}} X_{k} M_{f^{0}}\right)_{i t}\right|^{26}$, $\mathbb{E}\left|\left(M_{\lambda^{0}} X_{k}\right)_{i t}\right|^{8}$, and $\mathbb{E}\left|\left(X_{k} M_{f^{0}}\right)_{i t}\right|^{8}$ are bounded uniformly over $k, i, j, N$, and $T$.

(ii) Errors are i.i.d. Normal: The error matrix $e$ is independent of $\lambda^{0}, f^{0}, \bar{X}_{k}$, and $\widetilde{X}_{k}^{\mathrm{str}}, k=1, \ldots, K$, and its elements $e_{i t}$ are independent and identically distributed as $\mathcal{N}\left(0, \sigma^{2}\right)$ across $i$ and over $t$.

(iii) Number of Factors not Underestimated: We have $R \geq R^{0}:=$ $\operatorname{rank}\left(\lambda^{0} f^{0 \prime}\right)$.

REMARKS: (i) Assumption SF imposes that the factor $f^{0}$ and the factor loading $\lambda^{0}$ are strong. The strong factor assumption is regularly imposed in the literature on large $N$ and $T$ factor models, including Bai and Ng (2002), Stock and Watson (2002), and Bai (2009a).

(ii) Assumption $\mathrm{NC}$ demands that there exists significant sampling variation in the regressors after concentrating out $R+R^{0}$ factors (or factor loadings). It is a sample version of the identification Assumption ID(iii) and it is

the numerical evaluation of $\mathcal{L}_{N T}^{R}(\beta)$ is unproblematic for moderately large values of $T$. Since the model is symmetric under $N \leftrightarrow T, \Lambda \leftrightarrow F, Y \leftrightarrow Y^{\prime}$, and $X_{k} \leftrightarrow X_{k}^{\prime}$, there also exists a dual formulation of $\mathcal{L}_{N T}^{R}(\beta)$ that involves solving an eigenvalue problem for an $N \times N$ matrix. 
essentially equivalent to Assumption A of Bai (2009a), but avoids mentioning the unobserved loadings $\lambda^{0}{ }^{15}$

(iii) Assumption NC is violated if there exists a linear combination $\alpha \cdot X$ of the regressors with $\alpha \neq 0$ and $\operatorname{rank}(\alpha \cdot X) \leq R+R^{0}$, that is, the assumption rules out low-rank regressors like time-invariant regressors or cross-sectionally invariant regressors. These low-rank regressors require a special treatment in the interactive fixed effect model (see Bai (2009a) and Moon and Weidner (2014)) and we do not consider them in the present paper. If one is not interested explicitly in their regression coefficients, then one can always eliminate the low-rank regressors by an appropriate projection of the data, for example, subtraction of the time (or cross-sectional) means from the data eliminates all time-invariant (or cross-sectionally invariant) regressors; see Section 5 for an example of this.

(iv) The norm restriction in Assumption LL(i)(b) is a high-level assumption. It is satisfied as long as $\widetilde{X}_{k, i t}^{\text {str }}$ is mean zero and weakly correlated across $i$ and over $t$; for details, see Appendix A.1 and Lemma A.1 there.

(v) Assumption LL(i) imposes that each regressor consists of three parts: (a) a strictly exogenous low-rank component, (b) a strictly exogenous component satisfying a norm restriction, and (c) a weakly exogenous component that follows a linear process with innovation given by the lagged error term $e_{i t}$. For example, if $X_{k, i t} \sim$ i.i.d. $\mathcal{N}\left(\mu_{k}, \sigma_{k}^{2}\right)$, independent of $e$, then we have $\bar{X}_{k, i t}=\mu_{k}$, $\widetilde{X}_{k, i t}^{\text {str }} \sim$ i.i.d. $\mathcal{N}\left(0, \sigma_{k}^{2}\right)$, and $\widetilde{X}_{k}^{\text {weak }}=0$. Assumption LL(i) is also satisfied for a stationary panel vector autoregression (VAR) with interactive fixed effects as in Holtz-Eakin, Newey, and Rosen (1988). A special case of this is a dynamic panel regression with fixed effects, where $Y_{i t}=\beta Y_{i, t-1}+\lambda_{i}^{0 \prime} f_{t}^{0}+e_{i t}$, with $|\beta|<1$ and "infinite history." In this case, we have $X_{i t}=Y_{i, t-1}=\bar{X}_{i t}+$ $\widetilde{X}_{i t}^{\text {str }}+\widetilde{X}_{i t}^{\text {weak }}$, where $\bar{X}_{i t}=\lambda_{i}^{0 \prime} \sum_{\tau=1}^{\infty} \beta^{\tau-1} f_{t-\tau}^{0}, \widetilde{X}_{i t}^{\text {str }}=\sum_{\tau=t}^{\infty} \beta^{\tau-1} e_{i, t-\tau}$, and $\widetilde{X}_{i t}^{\text {weak }}=$ $\sum_{\tau=0}^{t-1} \beta^{\tau-1} e_{i, t-\tau}$.

(vi) Assumption LL(i) is more restrictive than Assumption 5 in Moon and Weidner (2014), where $R^{0}$ is assumed to be known. However, it is more general than the restriction on the regressors in Pesaran (2006), where-in our notation-the decomposition $X_{k}=\bar{X}_{k}+\widetilde{X}_{k}^{\text {str }}$ is imposed, but the lower rank component $\bar{X}_{k}$ needs to satisfy further assumptions, and the weakly exogenous component $\widetilde{X}_{k}^{\text {weak }}$ is not considered. Bai (2009a) requires no such decomposition, but imposes strict exogeneity of the regressors.

\footnotetext{
${ }^{15}$ By dropping the expected value from Assumption ID(iii) and replacing the zero lower bound by a positive constant, one obtains $\inf _{F}\left[x^{\prime}\left(M_{F} \otimes M_{\lambda^{0}}\right) x /(N T)\right] \geq b>0$ w.p.a.1, which is equivalent to Assumption A of Bai (2009a) and can also be rewritten as $\min _{\|\alpha\|=1} \inf _{F} \operatorname{Tr}\left[M_{\lambda^{0}}(\alpha \cdot X)^{\prime} M_{F}(\alpha\right.$. $X) /(N T)] \geq b$. A slightly stronger version of the assumption, which avoids mentioning the unobserved factor loading $\lambda^{0}$, reads $\min _{\|\alpha\|=1} \inf _{F} \inf _{\lambda} \operatorname{Tr}\left[M_{\lambda}(\alpha \cdot X)^{\prime} M_{F}(\alpha \cdot X) /(N T)\right] \geq b$, where $F \in \mathbb{R}^{T \times R}$ and $\lambda \in \mathbb{R}^{N \times R^{0}}$, and this slightly stronger version is equivalent to Assumption NC.
} 
(vii) Among the conditions in Assumption LL, the i.i.d. normality condition in Assumption LL(ii) may be the most restrictive. In Appendix A.4, we provide an alternative version of Theorem 3.1 that imposes more general high-level conditions. Verifying those high-level conditions requires results on the eigenvalues and eigenvectors of random covariance matrices, which can be verified for i.i.d. normal errors by using known results from the random matrix theory literature; see Section 4.3 for more details. We believe, however, that those high-level conditions and thus our main result hold more generally, and we explore nonnormal and serially correlated errors in our Monte Carlo simulations below.

THEOREM 3.1-Main Result: Let Assumptions SF, NC, and LL hold, and consider a limit $N, T \rightarrow \infty$ with $N / T \rightarrow \kappa^{2}, 0<\kappa<\infty$. Then we have

$$
\sqrt{N T}\left(\widehat{\beta}_{R}-\beta^{0}\right)=\sqrt{N T}\left(\widehat{\beta}_{R^{0}}-\beta^{0}\right)+o_{P}(1) .
$$

Theorem 3.1 follows from Theorem A.3 and Lemma A.4 in the Appendix, whose proof is given in the Supplemental Material. The theorem guarantees that the asymptotic distribution of $\widehat{\beta}_{R}, R \geq R^{0}$, is identical to that of $\widehat{\beta}_{R^{0}}$ in (3.4) below.

The limiting distribution of $\sqrt{N T}\left(\widehat{\beta}_{R^{0}}-\beta^{0}\right)$ with known $R^{0}$ is available in the existing literature. According to Bai (2009a) and Moon and Weidner (2014),

$$
\sqrt{N T}\left(\widehat{\beta}_{R^{0}}-\beta^{0}\right) \Rightarrow \mathcal{N}\left(-\kappa \operatorname{plim} W^{-1} B, \sigma^{2} \operatorname{plim} W^{-1}\right),
$$

where $W$ is the $K \times K$ matrix with elements $W_{k_{1} k_{2}}=\frac{1}{N T} \operatorname{Tr}\left(M_{\lambda^{0}} X_{k_{1}} M_{f^{0}} X_{k_{2}}^{\prime}\right)$ and $B$ is the $K$-vector with elements $B_{k}=\frac{1}{N} \operatorname{Tr}\left[P_{f 0} \mathbb{E}\left(e^{\prime} X_{k}\right)\right] \cdot{ }^{16}$

The result (3.4) holds under the assumptions of Theorem 3.1 and also assuming that $\operatorname{plim} W^{-1} B$ and plim $W^{-1}$ exist, where plim refers to the probability limit as $N, T \rightarrow \infty$. Note that Assumption NC guarantees that $W$ is invertible asymptotically. The asymptotic bias in (3.4) is an incidental parameter bias due to predetermined regressors and is equal to zero for strictly exogenous regressors (for which $\left.\mathbb{E}\left(e^{\prime} X_{k}\right)=0\right)$; it generalizes the well known Nickell (1981) bias of the within-group estimator for dynamic panel models.

\footnotetext{
${ }^{16}$ The asymptotic distribution in (3.4) can also be derived from Corollary 4.3 below under more general conditions than in Assumption LL (see Moon and Weidner (2014) for details). Here we have used the homoscedasticity of $e_{i t}$ to simplify the structure of the asymptotic variance and bias. Bai (2009a) finds further asymptotic bias in $\widehat{\beta}_{R^{0}}$ due to heteroscedasticity and correlation in $e_{i t}$, which in our asymptotic result is ruled out by Assumption LL(ii), but is studied in our subsequent Monte Carlo simulations. Moon and Weidner (2014) work out the additional asymptotic bias in $\widehat{\beta}_{R^{0}}$ due to predetermined regressors, which is allowed for in Theorem 3.1.
} 
Estimators for $\sigma^{2}, W$, and $B$ are given by ${ }^{17}$

$$
\begin{aligned}
& \widehat{\sigma}_{R}^{2}=\frac{1}{(N-R)(T-R)-K} \sum_{i=1}^{N} \sum_{t=1}^{T}\left(\widehat{e}_{R, i t}\right)^{2}, \\
& \widehat{W}_{R, k_{1} k_{2}}=\frac{1}{N T} \operatorname{Tr}\left(M_{\widehat{\Lambda}_{R}} X_{k_{1}} M_{\widehat{F}_{R}} X_{k_{2}}^{\prime}\right), \\
& \widehat{B}_{R, k}=\sum_{t=1}^{T} \sum_{\tau=t+1}^{t+M} P_{\widehat{F}_{R}, t \tau}\left[\frac{1}{N} \sum_{i=1}^{N} \widehat{e}_{R, i t} X_{k, i \tau}\right],
\end{aligned}
$$

where $\widehat{e}_{R, i t}$ denotes the $(i, t)$ th element of $\widehat{e}_{R}=Y-\widehat{\beta}_{R} \cdot X-\widehat{\Lambda}_{R} \widehat{F}_{R}^{\prime}, P_{\widehat{F}_{R}, t \tau}$ denotes the $(t, \tau)$ th element of $P_{\widehat{F}_{R}}=\mathbb{1}_{T}-M_{\widehat{F}_{R}}=\widehat{F}_{R}\left(\widehat{F}_{R}^{\prime} \widehat{F}_{R}\right)^{\dagger} \widehat{F}_{R}^{\prime}$, and $M \in$ $\{1,2,3, \ldots\}$ is a bandwidth parameter that also depends on the sample size $N, T$. Let $\widehat{W}_{R}$ and $\widehat{B}_{R}$ be the matrix and the vector with elements $\widehat{W}_{R, k_{1} k_{2}}$ and $\widehat{B}_{R, k}$, respectively.

The next theorem establishes the consistency of these estimators. Let $\lambda^{\text {red }} \in$ $\mathbb{R}^{N \times\left(R-R^{0}\right)}$ and $f^{\text {red }} \in \mathbb{R}^{T \times\left(R-R^{0}\right)}$ be the leading $R-R^{0}$ principal components obtained from the $N \times T$ matrix $M_{\lambda^{0}} e M_{f^{0}}$, that is, $\lambda^{\text {red }}$ and $f^{\text {red }}$ minimize the objective function $\left\|M_{\lambda^{0}} e M_{f^{0}}-\lambda^{\text {red }} f^{\text {red } /}\right\|_{\mathrm{HS}}^{2}$, analogous to $\widehat{\Lambda}_{R}$ and $\widehat{F}_{R}$ defined in (3.1). ${ }^{18}$

THEOREM 3.2-Consistency of Bias and Variance Estimators: (i) Let the conditions of Theorem 3.1 hold. Then we have $\left\|P_{\widehat{F}_{R}}-P_{\left[f^{0}, \text { fred }^{\mathrm{ra}}\right]}\right\|=o_{p}(1)$, $\left\|P_{\widehat{\Lambda}_{R}}-P_{\left[\lambda^{0}, \lambda^{\text {red }}\right]}\right\|=o_{p}(1), \widehat{\sigma}_{R}^{2}=\sigma^{2}+o_{P}(1)$, and $\widehat{W}_{R}=W+o_{P}(1)$.

(ii) In addition, let $X_{k, t}=\left(X_{k, 1 t}, \ldots, X_{k, N t}\right)^{\prime}$, and assume that (a) $\gamma_{\tau}$ in Assumption LL(i)(c) satisfies $\left|\gamma_{\tau}\right|<c \tau^{-d}$ for some $c>0$ and $d>1$, (b) $\left\|\lambda_{i}^{0}\right\|$ and $\left\|f_{t}^{0}\right\|$ are uniformly bounded over $i, t$ and $N, T$, (c) $\max _{t}\left\|X_{k, t}\right\|=$ $\mathcal{O}_{P}(\sqrt{N} \log N),{ }^{19}$ and $(\mathrm{d})$ the bandwidth $M \rightarrow \infty$ such that $M(\log T)^{2} T^{-1 / 6} \rightarrow 0$. Then we have $\widehat{B}_{R}=B+o_{P}(1)$.

Combining Theorems 3.1 and 3.2 and the asymptotic distribution in (3.4) allows inference on $\beta$ for $R \geq R^{0}$. In particular, the bias corrected estimator

${ }^{17}$ The first factor in $\widehat{\sigma}^{2}$ reflects the degree of freedom correction from estimating $\Lambda, F$, and $\beta$, but could simply be chosen as $1 / N T$ for the purpose of consistency. Note also that $P_{\widehat{F}_{R}, t \tau}=\mathcal{O}_{P}(1 / T)$, which explains why no $1 / T$ factor is required in the definition of $\widehat{B}_{R, k}$.

${ }^{18}$ The superscript "red" stands for redundant, because it turns out that $\lambda^{\text {red }}$ and $f^{\text {red }}$ are asymptotically close to the $R-R^{0}$ redundant principal components that are estimated in (3.1).

${ }^{19}$ The high-level assumption $\max _{t}\left\|X_{k, t}\right\|=\mathcal{O}_{P}(\sqrt{N} \log N)$ can be shown to be satisfied for the regressor component $\widetilde{X}_{k, i t}^{\text {weak }}$ above, and can be justified for the other regressor components, for example, by assuming that $\bar{X}_{k}$ and $\widetilde{X}_{k}^{\text {str }}$ are uniformly bounded. 


$$
\begin{aligned}
\widehat{\beta}_{R}^{\mathrm{BC}}=\widehat{\beta}_{R}+\frac{1}{T} \widehat{W}_{R}^{-1} \widehat{B}_{R} \text { satisfies }^{20} & \\
& \sqrt{N T}\left(\widehat{\beta}_{R}^{\mathrm{BC}}-\beta^{0}\right) \Rightarrow \mathcal{N}\left(0, \sigma^{2} W^{-1}\right) .
\end{aligned}
$$

\section{Heuristic Discussion of the Main Result}

Intuitively, the inclusion of unnecessary factors in the LS estimation is similar to the inclusion of irrelevant regressors in an ordinary least squares (OLS) regression. In the OLS case, it is well known that if those irrelevant extra regressors are uncorrelated with the regressors of interest, then they have no effect on the asymptotic distribution of the regression coefficients of interest. It is, therefore, natural to expect that if the extra estimated factors in $\widehat{F}_{R}$ are asymptotically uncorrelated with the regressors, then the result of Theorem 3.1 should hold. To explore this, remember that $\widehat{F}_{R}$ is given by the first $R$ principal components of the matrix $\left(Y-\widehat{\beta}_{R} \cdot X\right)^{\prime}\left(Y-\widehat{\beta}_{R} \cdot X\right)$, and write

$$
Y-\widehat{\beta}_{R} \cdot X=\lambda^{0} f^{0 \prime}+e-\left(\widehat{\beta}_{R}-\beta^{0}\right) \cdot X .
$$

The strong factor assumption and the consistency of $\widehat{\beta}_{R}$ guarantee that the first $R^{0}$ principal components of $\left(Y-\widehat{\beta}_{R} \cdot X\right)^{\prime}\left(Y-\widehat{\beta}_{R} \cdot X\right)$ are close to $f^{0}$ asymptotically, that is, the true factors are correctly picked up by the principal component estimator. The additional $R-R^{0}$ principal components that are estimated for $R>R^{0}$ cannot pick up anymore true factors and are thus mostly determined by the remaining term $e-\left(\widehat{\beta}_{R}-\beta^{0}\right) \cdot X$. The key question for the properties of the extra estimated factors, and thus of $\widehat{\beta}_{R}$, is therefore whether the principal components obtained from $e-\left(\widehat{\beta}_{R}-\beta^{0}\right) \cdot X$ are dominated by $e$ or by $\left(\widehat{\beta}_{R}-\beta^{0}\right) \cdot X$. Only if they are dominated by $e$ can we expect the extra factors in $\widehat{F}_{R}$ to be uncorrelated with $X$ and, thus, the result in Theorem 3.1 to hold. The result on $P_{\widehat{F}_{R}}$ in Theorem 3.2 shows that the additional estimated factors are indeed close to $f^{\text {red }}$, that is, are mostly determined by $e$, but this result is far from obvious a priori, as the following discussion shows.

Under our assumptions, we have $\|e\|=\mathcal{O}_{P}(\sqrt{N})$ and $\left\|X_{k}\right\|=\mathcal{O}_{P}(\sqrt{N T})$ as $N$ and $T$ grow at the same rate. Thus, if the convergence rate of $\widehat{\beta}_{R}$ is faster than $\sqrt{N}$, that is, $\left\|\widehat{\beta}_{R}-\beta^{0}\right\|=o_{P}(\sqrt{N})$, then we have $\|e\| \gg\left\|\left(\widehat{\beta}_{R}-\beta^{0}\right) \cdot X\right\|$ asymptotically and we expect the extra $\widehat{F}_{R}$ to be dominated by $e$. A crucial step in the derivation of Theorem 3.1 is therefore to show faster than $\sqrt{N}$ convergence of $\widehat{\beta}_{R}$. Conversely, we expect counterexamples to the main result to be such that the convergence rate of the estimator $\widehat{\beta}_{R}$ is not faster than $\sqrt{N}$,

\footnotetext{
${ }^{20}$ Instead of estimating the bias analytically, one can use the result that the bias is of order $T^{-1}$ and perform split panel bias correction as in Dhaene and Jochmans (2015), who instead of the conditions of Theorem 3.2(ii), only requires some stationary condition over time.
} 
and we provide such a counterexample-which, however, violates Assumptions LL-in Section 4.3 below. Whether the intuition about "inclusion of irrelevant regressors" carries over to the "inclusion of irrelevant factors" thus crucially depends on the convergence rate of $\widehat{\beta}_{R}$.

\section{ASYMPTOTIC THEORY AND DISCUSSION}

Here we introduce key intermediate results for the proof of the main theorem, Theorem 3.1, stated above. These intermediate results may be useful independently of the main result, for example, Moon and Weidner (2014) and Moon, Shum, and Weidner (2014) crucially use the results established in Section 4.2 for the case of known $R=R^{0}$. The assumptions introduced below are all implied by the low-level Assumptions LL above; see to Lemma A.4 in the Appendix.

\subsection{Consistency of $\widehat{\beta}_{R}$}

Here we present a consistency result for $\widehat{\beta}_{R}$ under an arbitrary asymptotic $N, T \rightarrow \infty$, that is, without the assumption that $N$ and $T$ grow at the same rate, which is imposed everywhere else in the paper. In addition to Assumption NC, we require the following high-level assumptions to obtain the result.

ASSUMPTION SN-Spectral Norm of $X_{k}$ and $e$ :

(i) We have $\left\|X_{k}\right\|=\mathcal{O}_{P}(\sqrt{N T}), k=1, \ldots, K$.

(ii) We have $\|e\|=\mathcal{O}_{P}(\sqrt{\max (N, T)})$.

AssumPTION EX-Weak Exogeneity of $X_{k}$ : We have $\frac{1}{\sqrt{N T}} \operatorname{Tr}\left(X_{k} e^{\prime}\right)=\mathcal{O}_{P}(1)$, $k=1, \ldots, K$.

THEOREM 4.1: Let Assumptions SN, EX, and NC be satisfied, and let $R \geq R^{0}$. For $N, T \rightarrow \infty$, we then have $\sqrt{\min (N, T)}\left(\widehat{\beta}_{R}-\beta^{0}\right)=\mathcal{O}_{P}(1)$.

REMARKS: (i) One can justify Assumption SN(i) by use of the norm inequality $\left\|X_{k}\right\| \leq\left\|X_{k}\right\|_{\mathrm{HS}}$ and the fact that $\left\|X_{k}\right\|_{\mathrm{HS}}^{2}=\sum_{i, t} X_{k, i t}^{2}=\mathcal{O}_{P}(N T)$, where the last step follows, for example, if $X_{k, i t}$ has a uniformly bounded second moment.

(ii) Assumption $\mathrm{SN}(\mathrm{ii})$ is a condition on the largest eigenvalue of the random covariance matrix $e^{\prime} e$, which is often studied in the literature on random matrix theory (e.g., Geman (1980), Bai, Silverstein, and Yin (1988), Yin, Bai, and Krishnaiah (1988), and Silverstein (1989)). The results in Latala (2005) show that $\|e\|=\mathcal{O}_{P}(\sqrt{\max (N, T)})$ if $e$ has independent entries with mean zero and uniformly bounded fourth moment. Weak dependence of the entries $e_{i t}$ across $i$ and over $t$ is also permissible; see Appendix A.1. 
(iii) Assumption EX requires exogeneity of the regressors $X_{k}$, allowing for predetermined regressors, and some weak dependence of $X_{k, i t} e_{i t}$ across $i$ and over $t^{21}$

(iv) The theorem imposes no restriction at all on $f^{0}$ and $\lambda^{0}$, apart from the condition $R \geq \operatorname{rank}\left(\lambda^{0} f^{0 \prime}\right) .{ }^{22}$ In particular, the strong factor Assumption SF is not imposed here, that is, consistency of $\widehat{\beta}_{R}$ holds independently of whether the factors are strong, weak, or not present at all. This is an important robustness result, which is new in the literature.

(v) Under an asymptotic where $N$ and $T$ grow at the same rate, which is imposed everywhere else in the paper, Theorem 4.1 shows $\sqrt{N}$ (or equivalently $\sqrt{T}$ ) consistency of the estimator $\widehat{\beta}_{R}$. To prove the consistency, we do not use the argument of the standard consistency proof for an extremum estimator that is to apply a uniform law of large numbers to the sample objective function to find the limit function that is uniquely minimized at the true parameter. Deriving the uniform limit of the objective function $\mathcal{L}_{N T}^{R^{0}}(\beta)$ is difficult. In the proof that is available in the Supplemental Material, we find a lower bound of the objective function $\mathcal{L}_{N T}^{R^{0}}(\beta)$ that is quadratic in $\beta-\beta^{0}$ asymptotically and we establish the desired consistency, extending the consistency proof in Bai (2009a).

(vi) The $\sqrt{N}$ consistency of $\widehat{\beta}_{R}$ implies that the residuals $Y-\widehat{\beta}_{R} \cdot X$ will be asymptotically close to $\lambda^{0} f^{0 \prime}+e .^{23}$ This allows consistent estimation of $R^{0}$ under a strong factor Assumption SF by employing the known techniques on factor models without regressors (by applying, e.g., Bai and $\mathrm{Ng}$ (2002) to $\left.Y-\widehat{\beta}_{R} \cdot X\right)$, as also discussed in Bai $(2009 \mathrm{~b}) .{ }^{24}$

(vii) Having a consistent estimator for $R^{0}$, say $\widehat{R}$, one can calculate $\widehat{\beta}_{\widehat{R}}$, which will be asymptotically equal to $\widehat{\beta}_{R^{0}}$. In practice, however, the finite sample properties of the estimator $\widehat{\beta}_{\widehat{R}}$ crucially depend on the finite sample properties of $\widehat{R}$. Many recent papers have documented difficulties in obtaining reliable estimates for $R^{0}$ at the finite sample (see, e.g., the simulation results of Onatski (2010) and Ahn and Horenstein (2013)), and those difficulties are also illustrated by our empirical example in Section 5.

\subsection{Quadratic Approximation of $\mathcal{L}_{N T}^{0}(\beta)\left(:=\mathcal{L}_{N T}^{R^{0}}(\beta)\right)$}

To derive the limiting distribution of $\widehat{\beta}_{R}$, we study the asymptotic properties of the profile objective function $\mathcal{L}_{N T}^{R}(\beta)$ around $\beta^{0}$. The expression in (3.3) cannot easily be discussed by analytic means, since no explicit formula for the

\footnotetext{
${ }^{21}$ Note that $\frac{1}{\sqrt{N T}} \operatorname{Tr}\left(X_{k} e^{\prime}\right)=\frac{1}{\sqrt{N T}} \sum_{i} \sum_{t} X_{k, i t} e_{i t}$.

${ }^{22}$ This is the main reason why we use a slightly different noncollinearity Assumption NC, which avoids mentioning $\lambda^{0}$, compared to Bai (2009a).

${ }^{23}$ In the sense that $\left\|\left(Y-\widehat{\beta}_{R} \cdot X\right)-\left(\lambda^{0} f^{0 \prime}+e\right)\right\|=\left\|\left(\widehat{\beta}_{R}-\beta\right) \cdot X\right\|=\mathcal{O}_{P}(\sqrt{N})$.

${ }^{24} \mathrm{Bai}$ (2009b) does not prove the required consistency and convergence rate of $\widehat{\beta}_{R}$ for $R>R^{0}$.
} 
eigenvalues of a matrix is available. In particular, a standard Taylor expansion of $\mathcal{L}_{N T}^{R}(\beta)$ around $\beta^{0}$ cannot easily be derived. Here, we consider the case of known $R=R^{0}$ and we perform a joint expansion of the corresponding profile objective function $\mathcal{L}_{N T}^{0}(\beta)$ in the regression parameters $\beta$ and in the idiosyncratic error terms $e$. To perform this joint expansion, we apply the perturbation theory of linear operators (e.g., Kato (1980)). We thereby obtain an approximate quadratic expansion of $\mathcal{L}_{N T}^{0}(\beta)$ in $\beta$, which can be used to derive the first order asymptotic theory of the LS estimator $\widehat{\beta}_{R^{0}}$; see Appendix A.2 for details. In addition to the $K \times K$ matrix $W$ already defined in Section 3, we now also define

$$
\begin{aligned}
C_{k}^{(1)}= & \frac{1}{\sqrt{N T}} \operatorname{Tr}\left(M_{\lambda^{0}} X_{k} M_{f^{0}} e^{\prime}\right), \\
C_{k}^{(2)}= & -\frac{1}{\sqrt{N T}}\left[\operatorname{Tr}\left(e M_{f^{0}} e^{\prime} M_{\lambda^{0}} X_{k} f^{0}\left(f^{0 \prime} f^{0}\right)^{-1}\left(\lambda^{0 \prime} \lambda^{0}\right)^{-1} \lambda^{0 \prime}\right)\right. \\
& +\operatorname{Tr}\left(e^{\prime} M_{\lambda^{0}} e M_{f^{0}} X_{k}^{\prime} \lambda^{0}\left(\lambda^{0 \prime} \lambda^{0}\right)^{-1}\left(f^{0 \prime} f^{0}\right)^{-1} f^{0 \prime}\right) \\
& \left.+\operatorname{Tr}\left(e^{\prime} M_{\lambda^{0}} X_{k} M_{f^{0}} e^{\prime} \lambda^{0}\left(\lambda^{0 \prime} \lambda^{0}\right)^{-1}\left(f^{0 \prime} f^{0}\right)^{-1} f^{0 \prime}\right)\right] .
\end{aligned}
$$

Let $C^{(1)}$ and $C^{(2)}$ be the $K$-vectors with elements $C_{k}^{(1)}$ and $C_{k}^{(2)}$, respectively.

THEOREM 4.2: Let Assumptions SF and SN be satisfied. Suppose that $N, T \rightarrow \infty$ with $N / T \rightarrow \kappa^{2}, 0<\kappa<\infty$. Then we have

$$
\begin{aligned}
\mathcal{L}_{N T}^{0}(\beta)= & \mathcal{L}_{N T}^{0}\left(\beta^{0}\right)-\frac{2}{\sqrt{N T}}\left(\beta-\beta^{0}\right)^{\prime}\left(C^{(1)}+C^{(2)}\right) \\
& +\left(\beta-\beta^{0}\right)^{\prime} W\left(\beta-\beta^{0}\right)+\mathcal{L}_{N T}^{0, \text { rem }}(\beta),
\end{aligned}
$$

where the remainder term $\mathcal{L}_{N T}^{0, \text { rem }}(\beta)$ satisfies, for any sequence $c_{N T} \rightarrow 0$,

$$
\sup _{\left\{\beta:\left\|\beta-\beta^{0}\right\| \leq c_{N T}\right\}} \frac{\left|\mathcal{L}_{N T}^{0, \text { rem }}(\beta)\right|}{\left(1+\sqrt{N T}\left\|\beta-\beta^{0}\right\|\right)^{2}}=o_{p}\left(\frac{1}{N T}\right) .
$$

The bound on the remainder ${ }^{25}$ in Theorem 4.2 is such that it has no effect on the first order asymptotic theory of $\widehat{\beta}_{R^{0}}$, as stated in the following corollary (see also Andrews (1999)).

\footnotetext{
${ }^{25}$ The expansion in Theorem 4.2 contains a term that is linear in $\beta$ and linear in $e\left(C^{(1)}\right.$ term), a term that is linear in $\beta$ and quadratic in $e\left(C^{(2)}\right.$ term), and a term that is quadratic in $\beta$ ( $W$ term). All higher order terms of the expansion are contained in the remainder term $\mathcal{L}_{N T}^{0 \text {,rem }}(\beta)$.
} 
COROllary 4.3: Let Assumptions SF, SN, EX, and NC be satisfied. In the limit $N, T \rightarrow \infty$ with $N / T \rightarrow \kappa^{2}, 0<\kappa<\infty$, we then have $\sqrt{N T}\left(\widehat{\beta}_{R^{0}}-\beta^{0}\right)=$ $W^{-1}\left(C^{(1)}+C^{(2)}\right)+o_{P}\left(1+\left\|C^{(1)}\right\|\right)$. If we furthermore assume that $C^{(1)}=\mathcal{O}_{P}(1)$, then we obtain

$$
\sqrt{N T}\left(\widehat{\beta}_{R^{0}}-\beta^{0}\right)=W^{-1}\left(C^{(1)}+C^{(2)}\right)+o_{P}(1)=\mathcal{O}_{P}(1) .
$$

Note that our assumptions already guarantee $C^{(2)}=\mathcal{O}_{P}(1)$ and that $W$ is invertible with $W^{-1}=\mathcal{O}_{P}(1)$, so this need not be explicitly assumed in Corollary 4.3.

REMARKS: (i) More details on the expansion of $\mathcal{L}_{N T}^{0}(\beta)$ are provided in Appendix A.2 and the formal proofs can be found in Section S.2 of the Supplemental Material.

(ii) Corollary 4.3 allows to replicate the results in Bai (2009a) and Moon and Weidner (2014) on the asymptotic distribution of $\widehat{\beta}_{R^{0}}$, including the result in formula (3.4) above. ${ }^{26}$ The assumptions of the corollary do not restrict the regressors to be strictly exogenous and do not impose Assumption LL.

(iii) If one weakens Assumption $\mathrm{SN}(\mathrm{ii})$ to $\|e\|=o_{P}\left(N^{2 / 3}\right)$, then Theorem 4.2 still continues to hold. If $C^{(2)}=\mathcal{O}_{P}(1)$, then Corollary 4.3 also holds under this weaker condition on $\|e\|$.

\subsection{Remarks on Deriving the Convergence Rate and Asymptotic Distribution of $\widehat{\beta}_{R}$ for $R>R^{0}$}

\section{An Example That Motivates Stronger Restrictions}

The results in Bai (2009a) and Corollary 4.3 above show that under appropriate assumptions, the estimator $\widehat{\beta}_{R}$ is $\sqrt{N T}$ consistent for $R=R^{0}$. For $R>R^{0}$, we know from Theorem 4.1 that $\widehat{\beta}_{R}$ is $\sqrt{N}$ consistent as $N$ and $T$ grow at the same rate, but we have not yet shown faster than $\sqrt{N}$ converge of $\widehat{\beta}_{R}$ for $R>R^{0}$, which according to the heuristic discussion at the end of Section 3 , is a very important intermediate step to obtain our main result. ${ }^{27}$ However, one

${ }^{26}$ Let $\rho, D(\cdot), D_{0}, D_{Z}, B_{0}$, and $C_{0}$ be the notation used in Assumption A and Theorem 3 of Bai (2009a), and let Bai's assumptions be satisfied. Then our $\kappa, W, C^{(1)}$, and $C^{(2)}$ satisfy $\kappa=\rho^{-1 / 2}$, $W=D\left(f^{0}\right) \rightarrow_{p} D>0, C^{(1)} \rightarrow_{d} \mathcal{N}\left(0, D_{Z}\right)$, and $W^{-1} C^{(2)} \rightarrow_{p} \rho^{1 / 2} B_{0}+\rho^{-1 / 2} C_{0}$. Corollary 4.3 can, therefore, be used to replicate Theorem 3 in Bai (2009a). For more details and extensions of this, refer to Moon and Weidner (2014).

${ }^{27}$ One reason why $\widehat{\beta}_{R}$ might only converge at $\sqrt{N}$ rate, but not faster, is weak factors (both for $R>R^{0}$ and for $R=R^{0}$ ). A weak factor (see, e.g., Onatski $(2010,2012)$ and Chudik, Pesaran, and Tosetti (2011)) might not be picked up at all or might only be estimated very inaccurately by the principal components estimator $\widehat{F}_{R}$, in which case that factor is not properly accounted for in the LS estimation procedure. If this happens and the weak factor is correlated with the regressors, then there is some uncorrected weak endogeneity problem, and $\widehat{\beta}_{R}$ will only converge at $\sqrt{N}$ rate. We do not consider the issue of weak factors any further in this paper. 
might not obtain a faster than $\sqrt{N}$ convergence rate of $\widehat{\beta}_{R}$ for $R>R^{0}$ without imposing further restrictions, as the following example shows.

EXAMPLE: Let $R^{0}=0$ (no true factors) and $K=1$ (one regressor). The true model reads $Y_{i t}=\beta^{0} X_{i t}+e_{i t}$ and we consider the data generating process (DGP)

$$
X_{i t}=a \tilde{X}_{i t}+\lambda_{x, i} f_{x, t}, \quad e=\left(\mathbb{1}_{N}+c \frac{\lambda_{x} \lambda_{x}^{\prime}}{N}\right) u\left(\mathbb{1}_{T}+c \frac{f_{x} f_{x}^{\prime}}{T}\right),
$$

where $e$ and $u$ are $N \times T$ matrices with entries $e_{i t}$ and $u_{i t}$, respectively, $\lambda_{x}$ is an $N$-vector with entries $\lambda_{x, i}$, and $f_{x}$ is a $T$-vector with entries $f_{x, t}$. Let $\tilde{X}_{i t}$ and $u_{i t}$ be mutually independent i.i.d. standard normally distributed random variables. Let $\lambda_{x, i} \in \mathcal{B}$ and $f_{x, t} \in \mathcal{B}$ be nonrandom sequences with bounded range $\mathcal{B} \subset \mathbb{R}$ such that $\frac{1}{N} \sum_{i=1}^{N} \lambda_{x, i}^{2} \rightarrow 1$ and $\frac{1}{T} \sum_{t=1}^{T} f_{x, t}^{2} \rightarrow 1$ asymptotically. ${ }^{28}$ Consider $N, T \rightarrow \infty$ such that $N / T \rightarrow \kappa^{2}, 0<\kappa<\infty$, and let $0<a<(1 / 2)^{2 / 3} \min \left(\kappa^{2}, \kappa^{-2}\right)$ and $c \geq \frac{(2+\sqrt{2})(1+\kappa)\left(1+\sqrt{3} a^{-1 / 4}\right)}{\min (1, \kappa)\left[1 / 2-a^{3 / 2} \max \left(\kappa, \kappa^{-1}\right)\right]} .{ }^{29}$ For this DGP, one can show that $\widehat{\beta}_{1}$, the LS estimator with $R=1>R^{0}$, only converges at a rate of $\sqrt{N}$ to $\beta^{0}$, but not faster.

The proof of the last statement is provided in the Supplemental Material. The DGP in this example satisfies all the assumptions imposed in Corollary 4.3 to derive the limiting distribution of the LS estimator for $R=R^{0}$, including $\sqrt{N T}$ consistency of $\widehat{\beta}_{R}$ for $R=R^{0}$ (=0 in this example). It also satisfies all the regularity conditions imposed in Bai (2009a). ${ }^{30}$ The aspect that is special about this DGP is that $\lambda_{x}$ and $f_{x}$ feature both in $X_{i t}$ and in the second moment structure of $e_{i t}$. The heuristic discussion at the end of Section 3 provides some intuition as to why this can be problematic, because the leading principal components obtained from only the error matrix $e$ will have a strong sample correlation with $X_{i t}$ for this DGP.

\section{Faster Than $\sqrt{N}$ Convergence of $\widehat{\beta}_{R}$}

In Appendix A.3, we summarize our results on faster than $\sqrt{N}$ convergence of $\widehat{\beta}_{R}$ for $R \geq R^{0}$. The above example shows that this requires more restrictive assumptions than those imposed for the analysis of the case $R=R^{0}$ above,

\footnotetext{
${ }^{28}$ We could also allow $\lambda_{x}$ and $f_{x}$ to be random (but independent of $e$ and $\tilde{X}$ ), and we could let the range of $\mathcal{B}$ be unbounded. We only assume nonrandom $\lambda_{x}$ and $f_{x}$ to guarantee that the DGP satisfies Assumption D of Bai (2009a), namely that $X$ and $e$ are independent (otherwise we only have mean independence, i.e., $\mathbb{E}(e \mid X)=0)$. Similarly, we only assume bounded $\mathcal{B}$ to satisfy the restrictions on $e_{i t}$ imposed in Assumption C of Bai (2009a).

${ }^{29}$ The bounds on the constants $a$ and $c$ imposed here are sufficient, but not necessary for the result of no faster than $\sqrt{N}$ convergence of $\widehat{\beta}_{1}$. Simulation evidence suggests that this result holds for a much larger range of $a, c$ values.

${ }^{30}$ See Section S.9 in the Supplemental Material for details.
} 
but the assumptions that we impose for this intermediate result are still significantly weaker than the Assumption LL required for our main result; in particular, either cross-sectional correlation or time-serial correlation of $e_{i t}$ is still allowed.

In Appendix A.3, we also provide one set of assumptions (Assumption DX-2) for faster than $\sqrt{N}$ convergence such that no additional conditions on $e$ are required, but where the regressors are restricted to essentially be lagged dependent variables in an $\operatorname{AR}(p)$ model with factors.

\section{On the Role of the i.i.d. Normality of $e_{i t}$}

We establish the asymptotic equivalence of $\widehat{\beta}_{R}$ and $\widehat{\beta}_{R^{0}}$ in Theorem 3.1 by showing that the LS objective function $\mathcal{L}_{N T}^{R}(\beta)$ can, up to a constant, be uniformly well approximated by $\mathcal{L}_{N T}^{0}(\beta)$ in shrinking neighborhoods around the true parameter. For this, we need not only the faster than $\sqrt{N}$ convergence rate of $\widehat{\beta}_{R}$, but also require the Assumption EV in Appendix A.4. This is a high-level assumption on the eigenvalues and eigenvectors of the random covariance matrices $E E^{\prime}$ and $E^{\prime} E$, where $E=M_{\lambda^{0}} e M_{f^{0}}$. The assumption essentially requires the eigenvalues of those matrices to be sufficiently separated from each other, as well as the eigenvectors of those matrices to be sufficiently uncorrelated with the regressors $X_{k}$, and with $e P_{f^{0}}$ and $P_{\lambda^{0}} e$.

We use the i.i.d. normality of $e_{i t}$ to verify those high-level conditions in Section S.4.2 of the Supplemental Material. There are three reasons why we can currently only verify those conditions for i.i.d. normal errors:

(i) The random matrix theory literature studies the eigenvalues and eigenvectors of random covariance matrices of the form $e e^{\prime}$ and $e^{\prime} e$, while we have to deal with the additional projectors $M_{\lambda^{0}}$ and $M_{f^{0}}$ in the random covariance matrices. These additional projections stem from integrating out the true factors and factor loadings of the model. If the error distribution is i.i.d. normal, and independent from $\lambda^{0}$ and $f^{0}$, then these projections are unproblematic, since the distribution of $e$ is rotationally invariant from the left and the right in that case, so that the projections are mathematically equivalent to a reduction of the sample size by $R^{0}$ in both panel dimensions.

(ii) In the i.i.d. normal case, one can furthermore use the invariance of the distribution of $e$ under orthonormal rotations from the left and from the right to also fully characterize the distribution of the eigenvectors of $E E^{\prime}$ and $E E^{\prime}{ }^{31}$ The conjecture in the random matrix theory literature is that the limiting distribution of the eigenvectors of a random covariance matrix is "distribution free," that is, is independent of the particular distribution of $e_{i t}$; see, for example, Silverstein (1990) and Bai (1999). However, we are not currently aware of a formulation and corresponding proof of this conjecture that is sufficient

\footnotetext{
${ }^{31}$ Rotational invariance implies that the distribution of the normalized eigenvectors is given by the Haar measure of a rotation group manifold.
} 
for our purposes, that is, that would allow us to verify our high-level Assumption EV more generally.

(iii) We also require certain properties of the eigenvalues of $E E^{\prime}$ and $E E^{\prime}$. Eigenvalues are studied more intensely than eigenvectors in the random matrix theory literature, and it is well known that the properly normalized empirical distribution of the eigenvalues (the so-called empirical spectral distribution) of an i.i.d. sample covariance matrix converges to the Marčenko-Pastur law (Marčenko and Pastur (1967)) for asymptotics where $N$ and $T$ grow at the same rate. This result does not require normality, and results on the limiting spectral distribution are also known for non-i.i.d. matrices. However, to check our high-level Assumption EV, we also need results on the convergence rate of the empirical spectral distribution to its limit law, which is an ongoing research subject in the literature (e.g., Bai (1993), Bai, Miao, and Yao (2003), Götze and Tikhomirov (2010)), and we are currently only aware of results on this convergence rate for the case of either i.i.d. or i.i.d. normal errors. To verify the high-level assumption, we furthermore use a result from Johnstone (2001) and Soshnikov (2002) that shows that the properly normalized few largest eigenvalues of $E E^{\prime}$ and $E E^{\prime}$ converge to the Tracy-Widom law, and to our knowledge this result is not established for error distributions that are not i.i.d. normal.

In spite of these severe mathematical challenges, we believe that, in principle, our high-level Assumption EV could be verified for more general error distributions, implying that our main result of asymptotic equivalence of $\widehat{\beta}_{R}$ and $\widehat{\beta}_{R^{0}}$ holds more generally. This is also supported by our Monte Carlo simulations, where we explore nonindependent and nonnormal error distributions.

\section{EMPIRICAL ILLUSTRATION}

As an illustrative empirical example, we estimate the dynamic effects of unilateral divorce law reforms on the statewise divorce rates in the United States. The impact of the divorce law reform has been studied by many researchers (e.g., Allen (1992), Peters (1986, 1992), Gray (1998), Friedberg (1998), Wolfers (2006), and Kim and Oka (2014)). In this section, we revisit this topic, extending Wolfers (2006) and Kim and Oka (2014) by controlling for interactive fixed effects and also a lagged dependent variable.

Let $Y_{i t}$ denote the number of divorces per 1000 people in state $i$ at time $t$, and let $D_{i}$ denote the year in which state $i$ introduced the unilateral divorce law, that is, before year $D_{i}$, state $i$ had a consent divorce law, while from $D_{i}$ onward, state $i$ had a unilateral "no-fault" divorce law, which lowers the barrier for divorce. The goal is to estimate the dynamic effects of this law change on the divorce rate. The empirical model we estimate is

$$
Y_{i t}=\beta_{0} Y_{i, t-1}+\sum_{k=1}^{8} \beta_{k} X_{k, i t}+\alpha_{i}+\gamma_{i} t+\delta_{i} t^{2}+\mu_{t}+\lambda_{i}^{\prime} f_{t}+e_{i t},
$$


where we follow Wolfers (2006) in defining the regressors as biannual dummies:

$$
\begin{aligned}
& X_{k, i t}=\mathbb{1}\left\{D_{i}+2(k-1) \leq t \leq D_{i}+2 k-1\right\} \text { for } k=1, \ldots, 7, \\
& X_{8, i t}=\mathbb{1}\left\{D_{i}+2(k-1) \leq t\right\} .
\end{aligned}
$$

The dummy variable and quadratic trend specification $\alpha_{i}+\gamma_{i} t+\delta_{i} t^{2}+\mu_{t}$ is also used in Friedberg (1998) and Wolfers (2006). The additional interactive fixed effects $\lambda_{i}^{\prime} f_{t}$ were added in Kim and Oka (2014) to control for additional unobserved heterogeneity in the divorce rate, for example, due to social, cultural, or demographic factors. We extend the specification further by adding a lagged dependent variable $Y_{i, t-1}$ to control for state dependence of the divorce rate, but we also report results without $Y_{i, t-1}$ below. We use the data set of Kim and Oka (2014), ${ }^{32}$ which is a balanced panel of $N=48$ states over $T=33$ years, leaving $T=32$ time periods if the lagged dependent variable is included.

For estimation, we first eliminate $\alpha_{i}, \gamma_{i}, \delta_{i}$, and $\mu_{t}$ from the model by projecting the outcome variable and all regressors accordingly, for example, $\widetilde{Y}=M_{1_{N}} Y M_{\left(1_{T}, \mathbf{t}, \mathbf{t}^{2}\right)}$, where $1_{N}$ and $1_{T}$ are $N$ and $T$ vectors, respectively, with all entries equal to 1 , and $\mathbf{t}$ and $\mathbf{t}^{2}$ are $T$ vectors with entries $t$ and $t^{2}$, respectively. The model after projection reads $\widetilde{Y}_{i t}=\beta_{0} \widetilde{Y}_{i, t-1}+\sum_{k=1}^{8} \beta_{k} \widetilde{X}_{k, i t}+\widetilde{\lambda}_{i}^{\prime} \widetilde{f}_{t}+\widetilde{e}_{i t}$, which is exactly the model we have studied so far in this paper. ${ }^{33}$ We use the LS estimator described above to estimate this model. The projection reduces the effective sample size to $N=48-1=47$ and $T=32-3=29$, which should be accounted for when calculating standard errors, for example, in the formula for $\widehat{\sigma}_{R}^{2}$ above (degrees of freedom correction). Our theoretical results are still applicable. ${ }^{34}$

We need to decide on a number of factors $R$ when implementing the LS estimator. As already mentioned in the last remark in Section 4.1, we can apply known techniques from the literature on factor models without regressors to obtain a consistent estimator of $R^{0}$. To do so, we choose a maximum number of factors of $R_{\max }=9$ to obtain the preliminary estimate $\widehat{\beta}_{R_{\max }}$ and then calculate the residuals $\widehat{u}_{i t}=\widetilde{Y}_{i t}-\widehat{\beta}_{R_{\max }, 0} \widetilde{Y}_{i, t-1}-\sum_{k=1}^{8} \widehat{\beta}_{R_{\max }, k} \widetilde{X}_{k, i t}$. We then apply the information criteria (IC), panel $C_{p}$ criteria (PC), and Bayes information criterion (BIC3) of Bai and $\mathrm{Ng}(2002),{ }^{35}$ the criterion described in Onatski

\footnotetext{
${ }^{32}$ The data are available from http://qed.econ.queensu.ca/jae/2014-v29.2/kim-oka/.

${ }^{33}$ To construct $\widetilde{Y}_{i, t-1}$, we first apply the lag operator and then apply the projections $M_{1_{N}}$ and $M_{\left(1_{T}, \mathbf{t}, \mathbf{t}^{2}\right)}$.

${ }^{34}$ If $e_{i t}$ is i.i.d. normal, then $\widetilde{e}_{i t}$ is not, but one can apply appropriate orthogonal rotations in $N$ and $T$ space such that $\widetilde{e}_{i t}$ becomes i.i.d. normal again, although with the sample size reduced to $N=47$ and $T=29$. The rotation has no effect on the LS estimator, that is, it does not matter whether we work in the original or the rotated frame.

${ }^{35}$ Following Onatski (2010) and Ahn and Horenstein (2013), we report only BIC3 among the Akaike information criterion (AIC) and BIC criteria of Bai and $\mathrm{Ng}(2002)$.
} 
TABLE I

ESTIMATED NUMBER OF FACTORS IN THE RESIDUALS $\widehat{u}$, USING DIFFERENT CRITERIA FOR ESTIMATION AND $R_{\max }=9^{\mathrm{a}}$

\begin{tabular}{llcccr}
\hline \hline Criterion & $\widehat{R}$ & Criterion & $\widehat{R}$ & Criterion & $\widehat{R}$ \\
\hline IC1 & 9 & PC1 & 9 & Onatski & 1 \\
IC2 & 7 & PC2 & 9 & ER & 1 \\
IC3 & 9 & PC3 & 9 & GR & 3 \\
BIC3 & 6 & & & & \\
\hline
\end{tabular}

${ }^{\mathrm{a}}$ The IC, PC, and BIC criteria are described in Bai and $\mathrm{Ng}$ (2002), the ER and GR criteria are from Ahn and Horenstein (2013), and we also use the criterion of Onatski (2010).

(2010), and the eigenvalue ratio (ER) and growth ratio (GR) criteria of Ahn and Horenstein (2013) to $\widehat{u} .{ }^{36}$ Most of these criteria also require specification of $R_{\max }$, and we continue to use $R_{\max }=9$. The corresponding estimation results for $R$ are presented in Table I. In addition, we also report the log scree plot, that is, the sorted eigenvalues of $\widehat{u} \cdot \widehat{u}$, in Figure 1 .

The log scree plot already shows that it is not obvious how to decompose the eigenvalue spectrum into a few larger eigenvalues stemming from factors and the remaining smaller eigenvalues stemming from the idiosyncratic error term. ${ }^{37}$ This problem is also reflected in the very different estimates for $R$ that

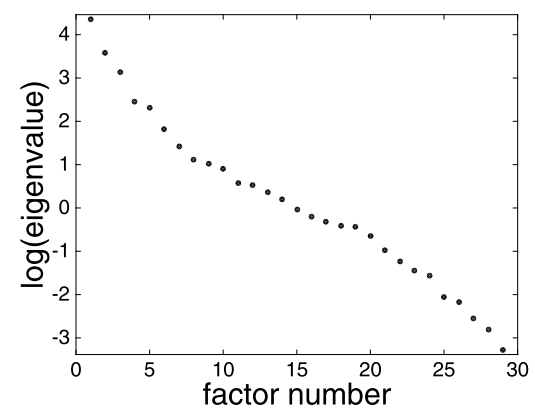

FIGURE 1.-Log scree plot. The natural logarithm of the sorted eigenvalues (corresponding to the principal components, or factors) of $\widehat{u}^{\prime} \widehat{u}$ are plotted.

\footnotetext{
${ }^{36}$ To include $R=0$ as a possible outcome for the Ahn and Horenstein (2013) criterion, we use the mock eigenvalue used in their simulations.

${ }^{37}$ The first largest eigenvalue is 2.2 times larger than the second eigenvalue, the second is 1.6 times larger than the third, and the third is 1.9 times larger than fourth. So the largest view eigenvalues are larger than the remaining ones, and the strong factor assumption might not be completely inappropriate here. However, deciding on a cutoff between factor and nonfactor eigenvalues is difficult.
} 
one obtains from the various criteria. It might appear that IC1, IC3, PC1, PC2, and PC3 all agree on $\widehat{R}=9$, but this is simply $\widehat{R}=R_{\max }$, and if we choose $R_{\max }=10$, then all these criteria deliver $\widehat{R}=10$, so this should not be considered a reliable estimate.

On the other hand, our asymptotic theory suggests that the exact choice of $R$ in the estimation of $\widehat{\beta}_{R}$ should not matter too much, as long as $R$ is chosen large enough to cover all relevant factors. Table II contains the estimation results for the bias corrected $\widehat{\beta}_{R}$ for $R \in\{0,1, \ldots, 9\}$. Table III contains estimates when the lagged dependent variable is not included in the model..$^{38}$ For all reported estimates, we perform bias correction and standard error estimation as described in Bai (2009a) and Moon and Weidner (2014). ${ }^{39}$

When ignoring the lagged dependent variable coefficient, one finds that in both Tables II and III, the estimation results for $\widehat{\beta}_{R}$ and the corresponding $t$ values are quite sensitive to changes in $R$ for very small values of $R$, but become much more stable as $R$ increases and actually do not change too much from roughly $R=2$ onward. These findings are very well in line with our asymptotic theory, and the dynamic effects of divorce law reform that we find are also similar to the findings in Wolfers (2006) and Kim and Oka (2014). The effect of the law reform on the divorce rates initially increases over time, is certainly significant in year 3-4 after the reform, and declines and becomes insignificant afterward. ${ }^{40}$

In contrast, the estimated coefficient on the lagged dependent variable in Table II is quite large and highly significant for small values of $R$, but decreases steadily with $R$ until it gets close to zero and is insignificant for $R \geq 8$. A plausible interpretation of this finding is that the model that includes the lagged dependent variable is misspecified, and that the estimated value of $\beta_{0}$ for small values of $R$ does not correspond to a true state dependence of $Y_{i t}$, but simply reflects the time-serial correlation of the error process being picked up by the

\footnotetext{
${ }^{38}$ The result for $R=7$ in Table III should be equal to column 6 in Table III of Kim and Oka (2014). The discrepancy is explained by a coding error in their bias computation. Note also that the result for $R=0$ in Table III does not match the one in Wolfers (2006), because he uses weighted least squares (WLS) with state population weights, while we use OLS for simplicity. Kim and Oka (2014) estimate both WLS and OLS, and find that the difference between the resulting estimates becomes insignificant once a sufficient number of interactive fixed effects are controlled for.

${ }^{39} \mathrm{We}$ correct for the biases due to heteroscedasticity in both panel dimensions worked out in Bai (2009a), as well as for the dynamic bias worked out in Moon and Weidner (2014). For the latter, we use the formula for $\widehat{B}_{R, k}$ above, with bandwidth $M=2$. For the standard error estimation, we allow for heteroscedasticity in both panel dimensions, also following Bai (2009a) and Moon and Weidner (2014). The bias and standard error formulas in those papers assume $R=R^{0}$ known, but we strongly expect that those formulas are robust toward $R>R^{0}$, as partly justified by Theorem 3.2. For the model without lagged dependent variable, we also allow for serial correlation in $e_{i t}$ when estimating the bias and standard deviation of $\widehat{\beta}_{R}$.

${ }^{40}$ The magnitude of the estimates is smaller than those in Wolfers (2006), that is, controlling for unobserved factors reduced the effect size, as already pointed out by Kim and Oka (2014).
} 
TABLE II

DyNAMiC EFFECTS OF DivorCE LAW REFORM ${ }^{\mathrm{a}}$

\begin{tabular}{|c|c|c|c|c|c|c|c|c|c|c|}
\hline & $R=0$ & $R=1$ & $R=2$ & $R=3$ & $R=4$ & $R=5$ & $R=6$ & $R=7$ & $R=8$ & $R=9$ \\
\hline \multirow[t]{2}{*}{ Lagged $Y$} & $0.432^{* *}$ & $0.623^{* *}$ & $0.573^{* *}$ & $0.411^{* *}$ & $0.369^{* *}$ & $0.191^{* *}$ & $0.137^{* *}$ & $0.154^{* *}$ & 0.063 & -0.026 \\
\hline & $(4.84)$ & $(15.38)$ & (13.81) & $(8.69)$ & $(8.19)$ & $(4.21)$ & $(2.93)$ & $(3.24)$ & (1.31) & $(-0.53)$ \\
\hline \multirow[t]{2}{*}{ Years 1-2 } & 0.043 & 0.089 & 0.098 & 0.105 & 0.112 & 0.043 & 0.087 & 0.064 & 0.089 & 0.039 \\
\hline & $(0.48)$ & (1.79) & (1.93) & $(1.80)$ & $(1.90)$ & $(0.70)$ & (1.45) & (1.08) & $(1.50)$ & $(0.68)$ \\
\hline \multirow[t]{2}{*}{ Years 3-4 } & 0.016 & $0.116^{*}$ & $0.147^{* *}$ & $0.214^{* *}$ & $0.242^{* *}$ & $0.170^{*}$ & $0.206^{*}$ & $0.162^{*}$ & $0.204^{*}$ & 0.149 \\
\hline & $(0.18)$ & $(2.15)$ & $(2.83)$ & $(3.31)$ & $(3.47)$ & $(2.21)$ & $(2.53)$ & (1.98) & $(2.41)$ & (1.61) \\
\hline Years 5-6 & -0.040 & 0.058 & 0.102 & $0.165^{*}$ & $0.183^{*}$ & 0.115 & 0.179 & 0.125 & 0.148 & $0.221^{*}$ \\
\hline \multirow[t]{2}{*}{ Years 7-8 } & -0.010 & 0.072 & 0.114 & 0.190 & 0.177 & 0.140 & 0.163 & 0.082 & 0.093 & 0.153 \\
\hline & $(-0.08)$ & $(0.80)$ & (1.19) & (1.64) & $(1.46)$ & (1.16) & (1.34) & $(0.67)$ & $(0.73)$ & $(1.15)$ \\
\hline \multirow[t]{2}{*}{ Years 9-10 } & -0.126 & 0.043 & 0.041 & 0.112 & 0.119 & 0.013 & 0.048 & -0.032 & 0.011 & 0.054 \\
\hline & $(-0.84)$ & $(0.40)$ & $(0.37)$ & $(0.86)$ & $(0.87)$ & $(0.09)$ & $(0.34)$ & $(-0.23)$ & $(0.08)$ & $(0.36)$ \\
\hline \multirow[t]{2}{*}{ Years 11-12 } & -0.122 & 0.088 & 0.062 & 0.122 & 0.109 & 0.000 & 0.042 & -0.018 & -0.015 & 0.025 \\
\hline & $(-0.71)$ & $(0.70)$ & $(0.48)$ & $(0.81)$ & $(0.69)$ & $(0.00)$ & $(0.25)$ & $(-0.11)$ & $(-0.09)$ & $(0.14)$ \\
\hline \multirow[t]{2}{*}{ Years 12-14 } & -0.122 & 0.163 & 0.097 & 0.143 & 0.109 & -0.029 & 0.017 & -0.032 & -0.045 & -0.040 \\
\hline & $(-0.59)$ & (1.09) & $(0.64)$ & $(0.83)$ & $(0.61)$ & $(-0.15)$ & $(0.08)$ & $(-0.17)$ & $(-0.24)$ & $(-0.21)$ \\
\hline
\end{tabular}

${ }^{\mathrm{a}}$ We report bias corrected LS estimates for the regression coefficients in model (5.1). Each column corresponds to a different number of factors $R \in\{0,1, \ldots, 9\}$ used in the estimation; $t$-values are reported in parentheses. 
TABLE III

SAME as TABle II, BUt Without INCLUding THE LAGGed DEPENDENT VARIABLE IN THE Model

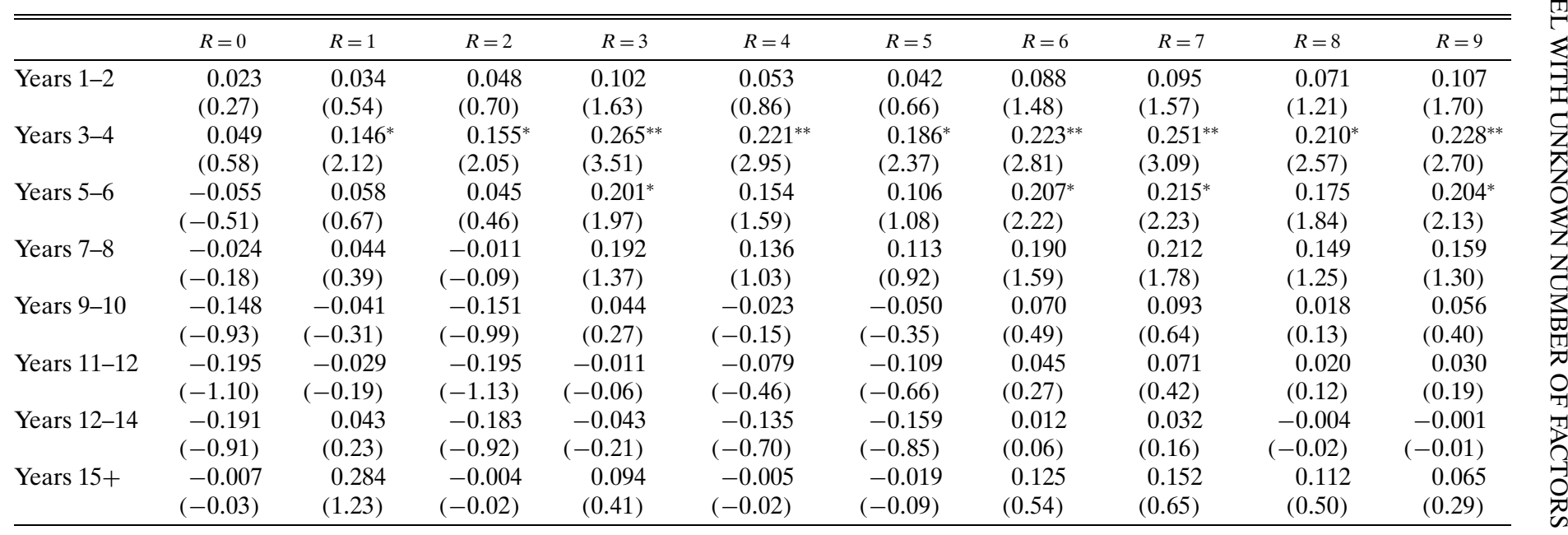


autoregressive model. According to this interpretation, once we include more and more factors into the model we control for more and more serial dependence of the unobserved error term, thus uncovering the true insignificance of $\beta_{0}$ in the estimates for $R \geq 8$.

This empirical example shows that instead of relying on a single estimate $\widehat{R}$ for the number of factors and reporting the corresponding $\widehat{\beta}_{\widehat{R}}$, it can be very informative to calculate $\widehat{\beta}_{R}$ for multiple values of $R$. Whether the estimated coefficients become stable for sufficiently large $R$ values, as our asymptotic theory suggests, is a useful robustness check for the model. When reporting the final results, then, it is better, within a reasonable range, to choose an $R$ that is too large than one that is too small.

We also perform a Monte Carlo simulation that is tailored toward the empirical application. For this, we use the static model without lagged dependent variable. To generate $Y_{i t}$ in equation (5.1) with $\beta_{0}=0$, we use the observed regressors $X_{k, i t}$, as described above, and as true parameters, we use the $\beta$ (bias corrected), $\alpha_{i}, \gamma_{i}, \delta_{i}, \mu_{t}, \lambda_{i}$, and $f_{t}$ obtained from the estimation with $R=4$ (i.e., $\beta_{k}$ as reported in the $R=4$ column of Table III). We generate $e_{i t}$ from an MA(1) model with $t(5)$ distributed innovations. Note that this error distribution violates Assumption LL(ii).

In this "empirical Monte Carlo," we have $N=48, T=33$, and true number of factors $R^{0}=4$. We find that the bias corrected estimates for $\beta_{k}, k=1, \ldots, 8$, are essentially unbiased when $R \geq R^{0}$ factors are used in the estimation, but for $R<R^{0}$, the coefficient estimates are often biased. For $\beta_{k}, k \geq 3$, there are only small changes in the standard deviation of the estimator between $R=4$ and $R=9$, but for $\beta_{k}, k=1,2$, we observe standard deviation inflation of up to $25 \%$ between $R=4$ and $R=9$. Given the relatively small sample size, the difference between $R=9$ and $R^{0}=4$ is relatively large, and some finite sample inefficiency is not too surprising. The detailed results are available in the Supplemental Material.

\section{MONTE CARLO SIMULATIONS}

In addition to the empirical Monte Carlo discussed above, we now investigate the finite sample properties of $\widehat{\beta}_{R}$ and $\widehat{\beta}_{R}^{\mathrm{BC}}$ further. In the simulations in this section, we use a generated regressor $X_{i t}$ that is correlated with the interactive fixed effects. The serial correlation of the error term $e_{i t}$ together with the data generating process (DGP) for $X_{i t}, \lambda_{i}$, and $f_{t}$ are such that the naive LS estimator has an asymptotic bias. This allows us to verify whether the bias is essentially unchanged for $R>R^{0}$ and whether bias correction works well for $R>R^{0}$ in finite samples. We also study various combinations of $N$ and $T$. 
The model is a static panel model with one regressor $(K=1)$, two factors $\left(R^{0}=2\right)$, and the DGP

$$
\begin{aligned}
& Y_{i t}=\beta^{0} X_{i t}+\sum_{r=1}^{2} \lambda_{i r}^{0} f_{t r}^{0}+e_{i t}, \\
& X_{i t}=1+\widetilde{X}_{i t}+\sum_{r=1}^{2}\left(\lambda_{i r}^{0}+\chi_{i r}\right)\left(f_{t r}^{0}+f_{t-1, r}^{0}\right), \\
& e_{i t}=\frac{1}{\sqrt{2}}\left(v_{i t}+v_{i, t-1}\right) .
\end{aligned}
$$

The random variables $\widetilde{X}_{i t}, \lambda_{i r}^{0}, f_{t r}^{0}, \chi_{i r}$, and $v_{i t}$ are mutually independent, with $\widetilde{X}_{i t}$ and $f_{t r}^{0} \sim$ i.i.d. $\mathcal{N}(0,1), \lambda_{i r}^{0}$ and $\chi_{i r} \sim$ i.i.d. $\mathcal{N}(1,1)$, and $v_{i t} \sim$ i.i.d. $t(5)$, that is, $v_{i t}$ has a Student's $t$-distribution with 5 degrees of freedom.

Note that this model satisfies Assumptions SF, NC, and LL(i), but not LL(ii). The error term $e_{i t}$ is not distributed as i.i.d. normal. The time series of $e_{i t}$ follows an MA(1) process with innovations distributed as $t(5)$.

We choose $\beta^{0}=1$, and use 10,000 repetitions in our simulation. The true number of factors is chosen to be $R^{0}=2$. For each draw of $Y$ and $X$, we compute the LS estimator $\widehat{\beta}_{R}$ according to equation (3.1) for different values of $R$, namely $R \in\{0,1,2,3,4,5\}$.

Table IV reports biases and standard deviations of the estimator $\widehat{\beta}_{R}$ for different combinations of $R, N$, and $T$. For $R<R^{0}=2$, the model is misspecified and $\widehat{\beta}_{R}$ turns out to be severely biased. There is also bias in $\widehat{\beta}_{R}$ for $R \geq R^{0}$, due to time-serial correlation of $e_{i t}$. This bias was worked out in Bai (2009a), which also discusses bias correction.

Table $\mathrm{V}$ reports various quantiles of the distribution of $\sqrt{N T}\left(\widehat{\beta}_{R}-\beta^{0}\right)$ for $N=T=100$ and $N=T=300$, and different values of $R \geq R^{0}$. From these tables, we see that as $N, T$ increases, the distribution of $\widehat{\beta}_{R}$ gets closer to that of $\widehat{\beta}_{R^{0}}$.

Table VI reports the size of a $t$-test with nominal size equal to $5 \%$ for $R \geq R^{0}$. We use the results in Bai (2009a) to correct for the leading $1 / N$ (not actually present in our DGP) and $1 / T$ (present in our DGP) biases in $\widehat{\beta}_{R}$ before calculating the $t$-test statistics, allowing for heteroscedasticity in both panel dimensions and for time-serial correlation when estimating the bias and standard deviation of $\widehat{\beta}_{R}$. The finite sample size distortions are mostly due to residual bias after bias correction, but also partly due to some finite sample downward bias in the standard error estimates. The size distortions increase with $R$, but for all values of $R \geq R^{0}$ in Table VI, the size distortions decrease rapidly as $T$ increases.

Monte Carlo simulation results for an AR(1) model with factors can be found in Section S.7 of the Supplemental Material. Those additional simulations show that the finite sample properties (e.g., for $T=30$ ) of $\widehat{\beta}_{R^{0}}$ and $\widehat{\beta}_{R}$, 
TABLE IV

For DifFERENT Combinations of SAMPLE Sizes $N$ AND $T$, We Report the Bias AND STANDARD DEVIATION OF THE ESTIMATOR $\widehat{\beta}_{R}$, FOR $R=0,1, \ldots, 5$, BASED ON SiMULATIONS With 10,000 RePetition of Design (6.1), WHERE THE TRUe Number of FACTORS Is $R^{0}=2$

\begin{tabular}{|c|c|c|c|c|c|c|c|c|}
\hline \multirow[b]{2}{*}{$R$} & \multicolumn{2}{|c|}{$T=10$} & \multicolumn{2}{|c|}{$T=30$} & \multicolumn{2}{|c|}{$T=100$} & \multicolumn{2}{|c|}{$T=300$} \\
\hline & Bias & SD & Bias & SD & Bias & SD & Bias & SD \\
\hline \multicolumn{9}{|c|}{$N=100$} \\
\hline 0 & 0.2286 & 0.0321 & 0.2301 & 0.0167 & 0.2305 & 0.0117 & 0.2305 & 0.0103 \\
\hline 1 & 0.1061 & 0.0552 & 0.1155 & 0.0296 & 0.1191 & 0.0195 & 0.1200 & 0.0160 \\
\hline 2 & -0.0385 & 0.0342 & -0.0166 & 0.0142 & -0.0053 & 0.0071 & -0.0019 & 0.0040 \\
\hline 3 & -0.0427 & 0.0342 & -0.0170 & 0.0142 & -0.0053 & 0.0072 & -0.0019 & 0.0040 \\
\hline 4 & -0.0450 & 0.0356 & -0.0172 & 0.0144 & -0.0053 & 0.0072 & -0.0019 & 0.0040 \\
\hline 5 & -0.0461 & 0.0370 & -0.0175 & 0.0146 & -0.0053 & 0.0073 & -0.0019 & 0.0041 \\
\hline \multicolumn{9}{|c|}{$N=300$} \\
\hline 0 & 0.2291 & 0.0298 & 0.2299 & 0.0136 & 0.2306 & 0.0082 & 0.2307 & 0.0065 \\
\hline 1 & 0.1054 & 0.0500 & 0.1159 & 0.0263 & 0.1193 & 0.0148 & 0.1203 & 0.0105 \\
\hline 2 & -0.0408 & 0.0237 & -0.0172 & 0.0085 & -0.0054 & 0.0041 & -0.0018 & 0.0023 \\
\hline 3 & -0.0442 & 0.0244 & -0.0175 & 0.0086 & -0.0054 & 0.0041 & -0.0018 & 0.0023 \\
\hline 4 & -0.0462 & 0.0258 & -0.0179 & 0.0087 & -0.0055 & 0.0041 & -0.0018 & 0.0023 \\
\hline 5 & -0.0468 & 0.0275 & -0.0182 & 0.0088 & -0.0055 & 0.0041 & -0.0018 & 0.0023 \\
\hline
\end{tabular}

$R>R^{0}$, can be quite different, but those differences vanish as $T$ becomes large, as predicted by our asymptotic theory. In general, we always expect some finite sample inefficiency from overestimating the number of factors.

TABLE V

QUANTILES OF THE DisTRIBUTION OF $\sqrt{N T}\left(\widehat{\beta}_{R}-\beta^{0}\right)^{\mathrm{a}}$

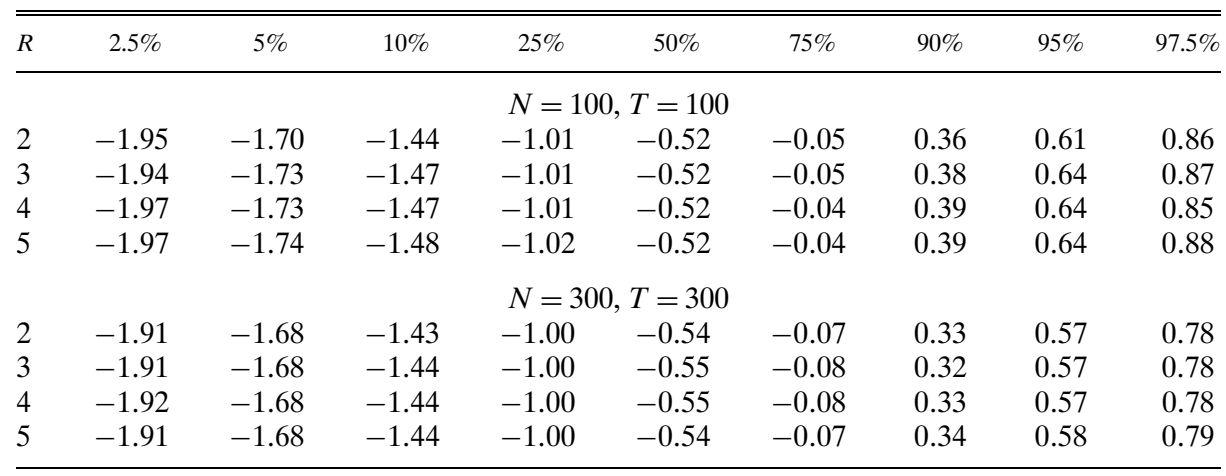

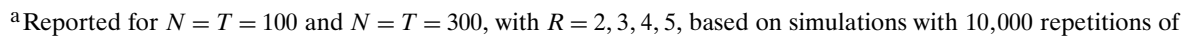
design (6.1), where the true number of factors is $R^{0}=2$.
} 
TABLE VI

The EMPIRICAL Size OF a $t$-TeSt With 5\% NOMINAL SizE ${ }^{a}$

\begin{tabular}{|c|c|c|c|c|c|c|c|c|}
\hline \multirow[b]{2}{*}{$R$} & \multicolumn{4}{|c|}{$N=100$} & \multicolumn{4}{|c|}{$N=300$} \\
\hline & $T=10$ & $T=30$ & $T=100$ & $T=300$ & $T=10$ & $T=30$ & $T=100$ & $T=300$ \\
\hline 2 & 0.252 & 0.084 & 0.057 & 0.051 & 0.535 & 0.146 & 0.055 & 0.051 \\
\hline 3 & 0.327 & 0.111 & 0.062 & 0.050 & 0.643 & 0.209 & 0.062 & 0.056 \\
\hline 4 & 0.358 & 0.141 & 0.067 & 0.054 & 0.672 & 0.280 & 0.070 & 0.057 \\
\hline 5 & 0.349 & 0.170 & 0.074 & 0.056 & 0.664 & 0.348 & 0.078 & 0.058 \\
\hline
\end{tabular}

${ }^{a}$ Reported for different combinations of $N, T$, and $R$, based on 10,000 repetitions of design (6.1). A bias corrected estimator $\widehat{\beta}_{R}^{\mathrm{BC}}$ is used to calculate the test statistics, and we allow for heteroscedasticity and time-serial correlation when estimating bias and standard deviation. Results for $R=0,1$ are not reported since those have size $=1$ due to misspecification.

\section{CONCLUSIONS}

We show that under certain assumptions, the limiting distribution of the LS estimator of a linear panel regression with interactive fixed effects does not change when we include redundant factors in the estimation. The implication of this is that one can use an upper bound of the number of factors $R$ in the estimation without asymptotic efficiency loss. However, some finite sample efficiency loss from overestimating $R$ is likely, so that $R$ should not be chosen too large in actual applications. We impose i.i.d. normality of the regression errors to derive the asymptotic result, because we require certain results on the eigenvalues and eigenvectors of random covariance matrices that are only known in that case. We expect that progress in the literature on large dimensional random covariance matrices will allow verification of our high-level assumptions under more general error distributions, and our Monte Carlo simulations suggest that the result also holds for nonnormal and correlated errors. We also provide multiple intermediate asymptotic results under more general conditions.

\section{APPENDIX}

\section{A.1. Spectral Norm of Random Matrices}

Consider an $N \times T$ matrix $u$ whose entries $u_{i t}$ have uniformly bounded second moments. Then we have $\|u\| \leq\|u\|_{\mathrm{HS}}=\sqrt{\sum_{i, t} u_{i t}^{2}}=\mathcal{O}_{P}(\sqrt{N T})$. However, in Assumptions LL(i)(b), DX-1(i), and DX-2(i), we impose $\left\|\widetilde{X}_{k}^{\text {str }}\right\|=\mathcal{O}_{P}\left(N^{3 / 4}\right)$ and $\left\|\widetilde{X}_{k}\right\|=\mathcal{O}_{P}\left(N^{3 / 4}\right)$, respectively, as $N$ and $T$ grow at the same rate, and in Assumption $\mathrm{SN}(\mathrm{ii})$, we impose $\|e\|=\mathcal{O}_{P}(\sqrt{\max (N, T)})$ under an arbitrary $\underset{\widetilde{X}_{k}}{\operatorname{asymptotic}} N, T \rightarrow \infty$. Those smaller asymptotic rates for the spectral norms of $\widetilde{X}_{k}^{\text {str }}, \widetilde{X}_{k}$, and $e$ can be justified by first assuming that the entries of these matrices are mean zero and have certain bounded moments, and, second, imposing weak cross-sectional and time-serial correlation. The purpose of this 
appendix section is to provide some examples of matrix distributions that make the last statement more precise. We consider the $N \times T$ matrix $u$, which can represent either $e, \widetilde{X}_{k}^{\text {str }}$, or $\widetilde{X}_{k}$.

EXAMPLE 1: If we assume that $\mathbb{E} u_{i t}=0$, that $\mathbb{E} u_{i t}^{4}$ is uniformly bounded, and that the $u_{i t}$ are independently distributed across $i$ and over $t$, then the results in Latala (2005) show that $\|u\|=\mathcal{O}_{P}(\sqrt{\max (N, T)})$.

EXAMPLE 2: Onatski (2013) provides the following example, which allows for both cross-sectional and time-serial dependence: Let $\epsilon$ be an $N \times T$ matrix with mean zero, independent entries that have uniformly bounded fourth moment, let $\epsilon_{t}$ denote the columns of $\epsilon$, and also define past $\epsilon_{t}, t \leq 0$, satisfying the same distributional assumptions. Let $u_{t}=\sum_{j=0}^{m} \Psi_{N, j} \epsilon_{t-j}$, where $m$ is a fixed integer, and $\Psi_{N, j}$ are $N \times N$ matrices such that $\max _{j}\left\|\Psi_{N, j}\right\|$ is uniformly bounded. Then the $N \times T$ matrix $u$ with columns $u_{t}$ satisfies $\|u\|=$ $\mathcal{O}_{P}(\sqrt{\max (N, T)})$.

More examples of matrix distributions that satisfy $\|u\|=\mathcal{O}_{P}(\sqrt{\max (N, T)})$ are discussed in Onatski (2013) and Moon and Weidner (2014). Theorem 5.48 and Remark 5.49 in Vershynin (2012) can also be used to obtain a slightly weaker bound on $\|u\|$ under very general correlation of $u$ in one of its dimensions.

Note that the random matrix theory literature often only discusses limits where $N$ and $T$ grow at the same rate and shows $\|u\|=\mathcal{O}_{P}(\sqrt{N})$ under that asymptotic. Those results can easily be extended to more general asymptotics with $N, T \rightarrow \infty$ by considering $u$ as a submatrix of a $\max (N, T) \times \max (N, T)$ matrix $u^{\text {big }}$ and using that $\|u\| \leq\left\|u^{\text {big }}\right\|$.

EXAMPLE 3: The following lemma provides a justification for the bounds on $\left\|\widetilde{X}_{k}^{\text {str }}\right\|$ and $\left\|\widetilde{X}_{k}\right\|$, allowing for a quite general type of correlation in both panel dimensions.

LEMMA A.1: Let $u$ be an $N \times T$ matrix with entries $u_{i t}$. Let $\Sigma_{i j}=$ $\frac{1}{T} \sum_{t=1}^{T} \mathbb{E}\left(u_{i t} u_{j t}\right)$ and let $\Sigma$ be the $N \times N$ matrix with entries $\Sigma_{i j}$. Let $\eta_{i j}=$ $\frac{1}{\sqrt{T}} \sum_{t=1}^{T}\left[u_{i t} u_{j t}-\mathbb{E}\left(u_{i t} u_{j t}\right)\right], \quad \Psi_{i j}=\frac{1}{N} \sum_{k=1}^{N} \mathbb{E}\left(\eta_{i k} \eta_{j k}\right), \quad$ and $\chi_{i j}=\frac{1}{\sqrt{N}} \times$ $\sum_{k=1}^{N}\left[\eta_{i k} \eta_{j k}-\mathbb{E}\left(\eta_{i k} \eta_{j k}\right)\right]$. Consider $N, T \rightarrow \infty$ such that $N / T$ converges to a finite positive constant, and assume that

(i) $\|\Sigma\|=\mathcal{O}(1)$,

(ii) $\frac{1}{N^{2}} \sum_{i, j=1}^{N} \mathbb{E}\left(\eta_{i j}^{2}\right)=\mathcal{O}(1)$,

(iii) $\frac{1}{N} \sum_{i, j=1}^{N} \Psi_{i j}^{2}=\mathcal{O}(1)$,

(iv) $\frac{1}{N^{2}} \sum_{i, j=1}^{N} \mathbb{E}\left(\chi_{i j}^{2}\right)=\mathcal{O}(1)$.

Then we have $\|u\|=\mathcal{O}_{P}\left(N^{5 / 8}\right)$. 
The lemma does not impose $\mathbb{E} u_{i t}=0$ explicitly, but justification of assumption (i) in the lemma usually requires $\mathbb{E} u_{i t}=0$. The assumptions (ii), (iii), and (iv) in the lemma can, for example, be justified by assuming appropriate mixing conditions in both panel dimensions; see, for example, Cox and Kim (1995) for the time-series case.

As pointed out above, our results in Section 4.2 can be obtained under the weaker condition $\|e\|=o_{P}\left(N^{2 / 3}\right)$, and then Lemma A.1 can also be applied with $u=e$. In that case, the assumptions in Lemma A.1 are not the same, but are similar to those imposed in Bai (2009a).

\section{A.2. Expansion of the Objective Function When $R=R^{0}$}

Here we provide a heuristic derivation of the expansion of $\mathcal{L}_{N T}^{0}(\beta)$ in Theorem 4.2. We expand the profile objective function $\mathcal{L}_{N T}^{0}(\beta)$ simultaneously in $\beta$ and in the spectral norm of $e$. Let the $K+1$ expansion parameters be defined by $\epsilon_{0}=\|e\| / \sqrt{N T}$ and $\epsilon_{k}=\beta_{k}^{0}-\beta_{k}, k=1, \ldots, K$, and define the $N \times T$ matrix $X_{0}=(\sqrt{N T} /\|e\|) e$. With these definitions, we obtain

$$
\begin{aligned}
\frac{1}{\sqrt{N T}}(Y-\beta \cdot X) & =\frac{1}{\sqrt{N T}}\left[\lambda^{0} f^{0 \prime}+\left(\beta^{0}-\beta\right) \cdot X+e\right] \\
& =\frac{\lambda^{0} f^{0 \prime}}{\sqrt{N T}}+\sum_{k=0}^{K} \epsilon_{k} \frac{X_{k}}{\sqrt{N T}} .
\end{aligned}
$$

According to equation (3.3) the profile objective function $\mathcal{L}_{N T}^{0}(\beta)$ can be written as the sum over the $T-R^{0}$ smallest eigenvalues of the matrix in (A.1) multiplied by its transposed. We consider $\sum_{k=0}^{K} \epsilon_{k} X_{k} / \sqrt{N T}$ as a small perturbation of the unperturbed matrix $\lambda^{0} f^{0 \prime} / \sqrt{N T}$, and thus expand $\mathcal{L}_{N T}^{0}(\beta)$ in the perturbation parameters $\epsilon=\left(\epsilon_{0}, \ldots, \epsilon_{K}\right)$ around $\epsilon=0$, namely

(A.2) $\quad \mathcal{L}_{N T}^{0}(\beta)$

$$
=\frac{1}{N T} \sum_{g=0}^{\infty} \sum_{k_{1}, \ldots, k_{g}=0}^{K} \epsilon_{k_{1}} \epsilon_{k_{2}} \cdots \epsilon_{k_{g}} L^{(g)}\left(\lambda^{0}, f^{0}, X_{k_{1}}, X_{k_{2}}, \ldots, X_{k_{g}}\right),
$$

where $L^{(g)}=L^{(g)}\left(\lambda^{0}, f^{0}, X_{k_{1}}, X_{k_{2}}, \ldots, X_{k_{g}}\right)$ are the expansion coefficients.

The unperturbed matrix $\lambda^{0} f^{0} / \sqrt{N T}$ has rank $R^{0}$, so that the $T-R^{0}$ smallest eigenvalues of the unperturbed $T \times T$ matrix $f^{0} \lambda^{0 \prime} \lambda^{0} f^{0 \prime} /(N T)$ are all zero, that is, $\mathcal{L}_{N T}^{0}(\beta)=0$ for $\epsilon=0$ and thus $L^{(0)}\left(\lambda^{0}, f^{0}\right)=0$. Due to Assumption SF, the $R^{0}$ nonzero eigenvalues of the unperturbed $T \times T$ matrix $f^{0} \lambda^{0 \prime} \lambda^{0} f^{0 \prime} /(N T)$ converge to positive constants as $N, T \rightarrow \infty$. This means that the "separating distance" of the $T-R^{0}$ zero eigenvalues of the unperturbed $T \times T$ matrix $f^{0} \lambda^{0 \prime} \lambda^{0} f^{0 \prime} /(N T)$ converges to a positive constant, that is, the next largest 
eigenvalue is well separated. This is exactly the technical condition under which the perturbation theory of linear operators guarantees that the above expansion of $\mathcal{L}_{N T}^{0}$ in $\epsilon$ exists and is convergent as long as the spectral norm of the perturbation $\sum_{k=0}^{K} \epsilon_{k} X_{k} / \sqrt{N T}$ is smaller than a particular convergence radius $r_{0}\left(\lambda^{0}, f^{0}\right)$, which is closely related to the separating distance of the zero eigenvalues. For details on that, see Kato (1980) and Section S.2 of the Supplemental Material, where we define $r_{0}\left(\lambda^{0}, f^{0}\right)$ and show that it converges to a positive constant as $N, T \rightarrow \infty$. Note that for the expansion (A.2), it is crucial that we have $R=R^{0}$, since the perturbation theory of linear operators describes the perturbation of the sum of all zero eigenvalues of the unperturbed matrix $f^{0} \lambda^{0 \prime} \lambda^{0} f^{0 \prime} /(N T)$. For $R>R^{0}$, the sum in $\mathcal{L}_{N T}^{R}(\beta)$ leaves out the $R-R^{0}$ largest of these perturbed zero eigenvalues, which results in a much more complicated mathematical problem, since the structure and ranking among these perturbed zero eigenvalues need to be discussed.

The above expansion of $\mathcal{L}_{N T}^{0}(\beta)$ is applicable whenever the operator norm of the perturbation matrix $\sum_{k=0}^{K} \epsilon_{k} X_{k} / \sqrt{N T}$ is smaller than $r_{0}\left(\lambda^{0}, f^{0}\right)$. Since our assumptions guarantee that $\left\|X_{k} / \sqrt{N T}\right\|=\mathcal{O}_{P}(1)$ for $k=0, \ldots, K$ and $\epsilon_{0}=$ $\mathcal{O}_{P}\left(\min (N, T)^{-1 / 2}\right)=o_{P}(1)$, we have $\left\|\sum_{k=0}^{K} \epsilon_{k} X_{k} / \sqrt{N T}\right\|=\mathcal{O}_{P}\left(\left\|\beta-\beta^{0}\right\|\right)+$ $o_{P}(1)$, that is, the above expansion is always applicable asymptotically within a shrinking neighborhood of $\beta^{0}$, which is sufficient since we already know that $\widehat{\beta}_{R}$ is consistent for $R \geq R^{0}$.

In addition, to guarantee convergence of the series expansion, the perturbation theory of linear operators also provides explicit formulas for the expansion coefficients $L^{(g)}$, namely for $g=1,2,3$, we have $L^{(1)}\left(\lambda^{0}, f^{0}, X_{k}\right)=0$, $L^{(2)}\left(\lambda^{0}, f^{0}, X_{k_{1}}, X_{k_{2}}\right)=\operatorname{Tr}\left(M_{\lambda^{0}} X_{k_{1}} M_{f^{0}} X_{k_{2}}^{\prime}\right)$, and $L^{(3)}\left(\lambda^{0}, f^{0}, X_{k_{1}}, X_{k_{2}}, X_{k_{3}}\right)=$ $-\frac{1}{3}\left[\operatorname{Tr}\left(M_{\lambda^{0}} X_{k_{1}} M_{f} X_{k_{2}}^{\prime} \lambda^{0}\left(\lambda^{0 \prime} \lambda^{0}\right)^{-1}\left(f^{0 \prime} f^{0}\right)^{-1} f^{0 \prime} X_{k_{3}}^{\prime}\right)+\cdots\right]$, where the dots refer to five additional terms obtained from the first one term by permutation of $k_{1}$, $k_{2}$, and $k_{3}$, so that the expression becomes totally symmetric in these indices. A general expression for the coefficients for all orders in $g$ is given in Lemma S.1 in the Supplemental Material. One can show that for $g \geq 3$, the coefficients $L^{(g)}$ are bounded as

$$
\begin{aligned}
& \frac{1}{N T}\left|L^{(g)}\left(\lambda^{0}, f^{0}, X_{k_{1}}, X_{k_{2}}, \ldots, X_{k_{g}}\right)\right| \\
& \quad \leq a_{N T}\left(b_{N T}\right)^{g} \frac{\left\|X_{k_{1}}\right\|}{\sqrt{N T}} \frac{\left\|X_{k_{2}}\right\|}{\sqrt{N T}} \cdots \frac{\left\|X_{k_{g}}\right\|}{\sqrt{N T}},
\end{aligned}
$$

where $a_{N T}$ and $b_{N T}$ are functions of $\lambda^{0}$ and $f^{0}$ that converge to finite positive constants in probability. This bound on the coefficients $L^{(g)}$ allows us to derive a bound on the remainder term when the profile objective expansion is truncated at a particular order. The expansion can be applied under more general asymptotics, but here we only consider the limit $N, T \rightarrow \infty$ with $N / T \rightarrow \kappa^{2}, 0<\kappa<\infty$, that is, $N$ and $T$ grow at the same rate. Then, 
apart from the constant $\mathcal{L}_{N T}^{0}\left(\beta^{0}\right)$, the relevant coefficients of the expansion, which are not treated as part of the remainder term, turn out to be $W_{k_{1} k_{2}}=$ $\frac{1}{N T} L^{(2)}\left(\lambda^{0}, f^{0}, X_{k_{1}}, X_{k_{2}}\right), C_{k}^{(1)}=\frac{1}{\sqrt{N T}} L^{(2)}\left(\lambda^{0}, f^{0}, X_{k}, e\right)=\frac{1}{\sqrt{N T}} \operatorname{Tr}\left(M_{\lambda^{0}} X_{k} M_{f^{0}} e^{\prime}\right)$, and $C_{k}^{(2)}=\frac{3}{2 \sqrt{N T}} L^{(3)}\left(\lambda^{0}, f^{0}, X_{k}, e, e\right)$, which corresponds exactly to the definitions in the main text. From the expansion (A.2) and the bound (A.3), we obtain Theorem 4.2. For a more rigorous derivation, we refer to Section S.2 in the Supplemental Material.

\section{A.3. $N^{3 / 4}$ Convergence Rate of $\widehat{\beta}_{R}$ for $R>R^{0}$}

The discussion at the end of Section 3 reveals that showing faster than $\sqrt{N}$ convergence of $\widehat{\beta}_{R}$ is a very important step on the way to the main result. For purely technical reasons, we show $N^{3 / 4}$ convergence first, but it will often be the case that if $\widehat{\beta}_{R}$ is $N^{3 / 4}$ consistent, then it is also $\sqrt{N T}$ consistent, as $N$ and $T$ grow at the same rate. We require one of the following two alternative assumptions.

ASSUMPTION DX-1-Decomposition of $X_{k}$ and Distribution of $e$, Version 1:

(i) For $k=1, \ldots, K$, we have $X_{k}=\bar{X}_{k}+\widetilde{X}_{k}$, where $\operatorname{rank}\left(\bar{X}_{k}\right)$ is bounded as $N, T \rightarrow \infty$, and $\left\|\bar{X}_{k}\right\|=\mathcal{O}_{P}(\sqrt{N T})$ and $\left\|\widetilde{X}_{k}\right\|=\mathcal{O}_{P}\left(N^{3 / 4}\right)$.

(ii) Let $u$ be an $N \times T$ matrix whose elements are distributed as i.i.d. $\mathcal{N}(0,1)$, independent of $\lambda^{0}, f^{0}$, and $\bar{X}_{k}, k=1, \ldots, K$, and let one of the following alternatives hold:

(a) We have e $=\Sigma^{1 / 2} u$, where $\Sigma$ is an $N \times N$ covariance matrix, independent of $u$, that satisfies $\|\Sigma\|=\mathcal{O}_{P}(1)$. In that case, define $g$ to be an $N \times Q$ matrix, independent of $u$, for some $Q \leq \sum_{k=1}^{K} \operatorname{rank}\left(\bar{X}_{k}\right)$, such that $g^{\prime} g=\mathbb{1}_{Q}$ and $\operatorname{span}\left(M_{\lambda^{0}} \bar{X}_{k}\right) \subset \operatorname{span}(g)$ for all $k=1, \ldots, K{ }^{41}$

(b) We have $e=u \Sigma^{1 / 2}$, where $\Sigma$ is a $T \times T$ covariance matrix, independent of $u$, that satisfies $\|\Sigma\|=\mathcal{O}_{P}(1)$. In that case, define $g$ to be a $T \times Q m a$ trix, independent of $u$, for some $Q \leq \sum_{k=1}^{K} \operatorname{rank}\left(\bar{X}_{k}\right)$, such that $g^{\prime} g=\mathbb{1}_{Q}$ and $\operatorname{span}\left(M_{f 0} \bar{X}_{k}^{\prime}\right) \subset \operatorname{span}(g)$ for all $k=1, \ldots, K$.

In addition, we assume that there exists a (potentially random) integer sequence $n=n_{N T}>0$ with $1 / n=\mathcal{O}_{P}(1 / N)$ such that $\mu_{n}(\Sigma) \geq\left\|g^{\prime} \Sigma g\right\|$. Finally, assume that either $R \geq Q$ or $g^{\prime} \Sigma g=\left\|g^{\prime} \Sigma g\right\| \mathbb{1}_{Q}+\mathcal{O}_{P}\left(N^{-1 / 2}\right)$.

AsSUMPTION DX-2-Decomposition of $X_{k}$ and Distribution of $e$, Version 2:

(i) For $k=1, \ldots, K$, we have $X_{k}=\bar{X}_{k}+\widetilde{X}_{k}$, such that $M_{\lambda^{0}} \bar{X}_{k} M_{f^{0}}=0$, and $\left\|\bar{X}_{k}\right\|=\mathcal{O}_{P}(\sqrt{N T})$ and $\left\|\tilde{X}_{k}\right\|=\mathcal{O}_{P}\left(N^{3 / 4}\right)$.

(ii) We have $\|e\|=\mathcal{O}_{P}(\sqrt{\max (N, T)})$ (same as Assumption $\mathrm{SN}(\mathrm{ii})$ ).

\footnotetext{
${ }^{41}$ The column space of $g$ thus contains the column space of all $M_{\lambda^{0}} \bar{X}_{k}$. The equality $g^{\prime} g=\mathbb{1}_{Q}$ is just a normalization.
} 
TheOREM A.2: Let $R>R^{0}$. Let Assumptions SF, NC, and EX hold, and let either Assumption DX-1 or Assumption DX-2 be satisfied. Consider $N, T \rightarrow \infty$ with $N / T \rightarrow \kappa^{2}, 0<\kappa<\infty$. Then we have $N^{3 / 4}\left(\widehat{\beta}_{R}-\beta^{0}\right)=\mathcal{O}_{P}(1)$.

REMARKS: (i) Assumption $\mathrm{SN}$ is not explicitly imposed in Theorem A.2, because it is already implied by both Assumption DX-1 and Assumption DX-2; see also Lemma A.4 below.

(ii) The restrictions that Assumption DX-1 imposes on $X_{k}$ are weaker than those imposed in Assumption LL above. The regressors are decomposed into a low-rank strictly exogenous part $\bar{X}_{k}$ and a term $\widetilde{X}_{k}$, which can be both strictly or weakly exogenous. The spectral norm bound $\left\|\widetilde{X}_{k}\right\|=\mathcal{O}_{P}\left(N^{3 / 4}\right)$ is satisfied as long as $\widetilde{X}_{k, i t}$ is mean zero and weakly correlated across $i$ and over $t$; see Appendix A.1. We can always write $\bar{X}_{k}=\ell h^{\prime}$ for some appropriate $\ell \in \mathbb{R}^{N \times \operatorname{rank}\left(\bar{X}_{k}\right)}$ and $h \in \mathbb{R}^{T \times \operatorname{rank}\left(\bar{X}_{k}\right)}$. Thus, the decomposition $X_{k}=\bar{X}_{k}+\widetilde{X}_{k}=\ell h^{\prime}+\widetilde{X}_{k}$ essentially imposes an approximate factor structure on $X_{k}$, with factor part $\bar{X}_{k}$ and idiosyncratic part $\widetilde{X}_{k}$. In addition to those conditions, we need sufficient variation in $X_{k}$, as formalized by the noncollinarity Assumption NC.

(iii) The restrictions that Assumption DX-1 imposes on $e$ are also weaker than those imposed in Assumption LL above. Normality is imposed, but either cross-sectional correlation and heteroscedasticity (case (a)) or time-serial correlation and heteroscedasticity (case (b)), described by $\Sigma$, are still allowed. The condition $\|\Sigma\|=\mathcal{O}_{P}(1)$ requires the correlation of $e_{i t}$ to be weak. ${ }^{42}$

(iv) The additional restrictions on $\Sigma$ in Assumption DX-1 rule out the type of correlation of the low-rank regressor part $\bar{X}_{k}$ with the second moment structure of $e_{i t}$ that was the key feature of the counterexample in Section 4.3. ${ }^{43}$ First, the condition $\mu_{n}(\Sigma) \geq\left\|g^{\prime} \Sigma g\right\|$ guarantees that the eigenvectors corresponding to the largest few eigenvectors of $\Sigma$ (the eigenvectors $\nu_{r}$ of $\Sigma$ when normalized satisfy $\left.\mu_{r}(\Sigma)=\nu_{r}^{\prime} \Sigma \nu_{r}\right)$ are not strongly correlated with $g$ (and thus with $\left.\bar{X}_{k}\right)$. Second, the condition $g^{\prime} \Sigma g=\left\|g^{\prime} \Sigma g\right\| \mathbb{1}_{Q}+\mathcal{O}_{P}\left(N^{-1 / 2}\right)$ guarantees that $\Sigma$ behaves almost as an identity matrix when projected with $g$, thus not possessing special structure in the "direction of $\bar{X}_{k}$." Both of these assumption are obviously satisfied when $\Sigma$ is proportional to the identity matrix.

(v) Instead of Assumption DX-1, we can also impose Assumption DX-2 to obtain $N^{3 / 4}$ consistency in Theorem A.2. The assumption on $e$ imposed in Assumption DX-2 is the same as in Assumption SN and, as already discussed above, this assumption is quite weak (see also Appendix A.1). However, Assumption DX-2 imposes a much stronger assumption on the regressors by requiring that $M_{\lambda^{0}} \bar{X}_{k} M_{f^{0}}=0$. This condition implies that $\bar{X}_{k}=\lambda^{0} h^{\prime}+\ell f^{0 \prime}$ for

\footnotetext{
${ }^{42} \mathrm{~A}$ sufficient condition for $\|\Sigma\|=\mathcal{O}_{P}(1)$ is, for example, $\max _{i} \sum_{j}\left|\Sigma_{i j}\right|=\mathcal{O}_{P}(1)$, formulated here for case (a). Note that $\Sigma$ is symmetric.

${ }^{43}$ However, in the example in Section 4.3, we have both time-serial and cross-sectional correlation in $e_{i t}$, one of which is already ruled out by Assumption DX-1.
} 
some $\ell \in \mathbb{R}^{N \times R^{0}}$ and $h \in \mathbb{R}^{T \times R^{0}}$, that is, the factor structure of the regressors is severely restricted. The AR(1) model discussed in Remark (v) in Section 3 does satisfy $M_{\lambda^{0}} \bar{X}_{k}=0$, and the same is true for a stationary $\operatorname{AR}(p)$ model without additional regressors, that is, for such $\operatorname{AR}(p)$ models with factors, we obtain $N^{3 / 4}$ consistency of $\widehat{\beta}_{R}$ without imposing strong assumptions (like normality) of $e_{i t}$. Assumption DX-2(i) is furthermore satisfied if $\bar{X}_{k}=0$, that is, if the regressors $X_{k}=\widetilde{X}_{k}$ satisfy $\left\|X_{k}\right\|=\mathcal{O}_{P}\left(N^{3 / 4}\right)$, which is true for zero mean weakly correlated processes (see Appendix A.1).

(vi) Theorem S.5 in the Supplemental Material provides an alternative $N^{3 / 4}$ consistency result, in which Assumptions DX-1 and DX-2 are replaced by a high-level condition, which is more general, but not easy to verify in terms of low-level assumptions.

\section{A.4. Asymptotic Equivalence of $\widehat{\beta}_{R^{0}}$ and $\widehat{\beta}_{R}$ for $R>R^{0}$}

Here, we provide high-level conditions on the singular values and singular vectors of the error matrix (or, equivalently, on the eigenvalues and eigenvectors of the corresponding random covariance matrix). Under those assumptions, we then establish the main result of the paper that $\widehat{\beta}_{R^{0}}$ and $\widehat{\beta}_{R}$ with $R>R^{0}$ are asymptotically equivalent, that is, $\sqrt{N T}\left(\widehat{\beta}_{R}-\widehat{\beta}_{R^{0}}\right)=o_{P}(1)$.

ASSUMPTION EV-Eigenvalues and Eigenvectors of a Random Cov Matrix: Let the singular value decomposition of $M_{\lambda^{0}} e M_{f^{0}}$ be given by $M_{\lambda^{0}} e M_{f^{0}}=$ $\sum_{r=1}^{Q} \sqrt{\rho_{r}} v_{r} w_{r}^{\prime}$, where $Q=\min (N, T)-R^{0}, \sqrt{\rho_{r}}$ are the singular values, and $v_{r}$ and $w_{r}$ are normalized $N$ - and T-vectors, respectively. ${ }^{44}$ Let $\rho_{1} \geq \rho_{2} \geq \cdots \geq$ $\rho_{Q} \geq 0$. We assume that there exists a constant $c>0$ and a series of integers $q_{N T}>R-R^{0}$ with $q_{N T}=o\left(N^{1 / 4}\right)$ such that as $N, T \rightarrow \infty$, we have

(i) $\frac{\rho_{R-R^{0}}}{N}>c$ w.p.a.1,

(ii) $\frac{1}{q_{N T}} \sum_{r=q_{N T}}^{Q}\left(\rho_{R-R^{0}}-\rho_{r}\right)^{-1}=\mathcal{O}_{P}(1)$,

(iii)

$$
\begin{aligned}
& \max _{r}\left\|v_{r}^{\prime} e P_{f^{0}}\right\|=o_{P}\left(N^{1 / 4} q_{N T}^{-1}\right), \quad \max _{r}\left\|w_{r}^{\prime} e^{\prime} P_{\lambda^{0}}\right\|=o_{P}\left(N^{1 / 4} q_{N T}^{-1}\right), \\
& \max _{r}\left\|v_{r}^{\prime} X_{k} P_{f^{0}}\right\|=o_{P}\left(N q_{N T}^{-1}\right), \quad \max _{r}\left\|w_{r}^{\prime} X_{k}^{\prime} P_{\lambda^{0}}\right\|=o_{P}\left(N q_{N T}^{-1}\right), \\
& \max _{r, s, k}\left|v_{r}^{\prime} X_{k} w_{s}\right|=o_{P}\left(N^{1 / 4} q_{N T}^{-1}\right),
\end{aligned}
$$

where $r, s=1, \ldots, Q$, and $k=1, \ldots, K$.

\footnotetext{
${ }^{44}$ Thus, $w_{r}$ is the normalized eigenvector corresponding to the eigenvalue $\rho_{r}$ of $M_{f^{0}} e^{\prime} M_{\lambda^{0}} e M_{f^{0}}$, while $v_{r}$ is the normalized eigenvector corresponding to the eigenvalue $\rho_{r}$ of $M_{\lambda^{0}} e M_{f^{0}} e^{\prime} M_{\lambda^{0}}$. We use a convention where eigenvalues with nontrivial multiplicity appear multiple times in the list of eigenvalues $\rho_{r}$, but under standard distributional assumptions on $e$ all eigenvalues are simple with probability 1 anyway.
} 
TheOrem A.3: Let $R>R^{0}$. Let Assumptions SF, NC, EX, and EV hold, let either Assumption DX-1 or Assumption DX-2 hold, and assume that $C^{(1)}=$ $\mathcal{O}_{P}(1)$. In the limit $N, T \rightarrow \infty$ with $N / T \rightarrow \kappa^{2}, 0<\kappa<\infty$, we then have

$$
\sqrt{N T}\left(\widehat{\beta}_{R}-\beta^{0}\right)=\sqrt{N T}\left(\widehat{\beta}_{R^{0}}-\beta^{0}\right)+o_{P}(1)=\mathcal{O}_{P}(1) .
$$

REMARKS: (i) Theorem A.3 also holds if we replace Assumptions EX, DX-1, and DX-2 by any other condition that guarantees that Assumption SN holds and that $N^{3 / 4}\left(\widehat{\beta}_{R}-\beta^{0}\right)=\mathcal{O}_{P}(1)$.

(ii) Consider Assumption EV(iii). Since $v_{r}$ and $w_{r}$ are the normalized singular vectors of $M_{\lambda^{0}} e M_{f^{0}}$, we expect them to be essentially uncorrelated with $X_{k}$ and $e P_{f^{0}}$, and, therefore, we expect $v_{r}^{\prime} X_{k} w_{s}=\mathcal{O}_{P}(1),\left\|v_{r}^{\prime} e P_{f^{0}}\right\|=$ $\mathcal{O}_{P}(1)$, and $\left\|w_{r}^{\prime} e^{\prime} P_{\lambda^{0}}\right\|=\mathcal{O}_{P}(1)$. We also expect $\left\|v_{r}^{\prime} X_{k} P_{f^{0}}\right\|=\mathcal{O}_{P}(\sqrt{T})$ and $\left\|w_{r}^{\prime} X_{k}^{\prime} P_{\lambda^{0}}\right\|=\mathcal{O}_{P}(\sqrt{N})$, which is different from the analogous expressions with $e$, since $X_{k}$ may be strongly correlated with $f^{0}$ and $\lambda^{0}$. The key to making this discussion rigorous is a good knowledge of the properties of the eigenvectors $v_{r}$ and $w_{r}$. If the entries $e_{i t}$ are i.i.d. normal, then the distribution of $v_{r}$ and $w_{r}$ can be characterized as follows: Let $\widetilde{v}$ be an $N$-vector with i.i.d. $\mathcal{N}(0,1)$ entries and let $\widetilde{w}$ be an $T$-vector with i.i.d. $\mathcal{N}(0,1)$ entries. Then we have $v_{r}={ }_{d}\left\|M_{\lambda^{0}} \widetilde{v}\right\|^{-1} M_{\lambda^{0}} \widetilde{v}$ and $w_{r}={ }_{d}\left\|M_{f^{0}} \widetilde{w}\right\|^{-1} M_{f^{0}} \widetilde{w}$; see also Lemma S.13 in the Supplemental Material. Here $={ }_{d}$ refers to "equal in distribution." Thus, if $R^{0}=0$, then $v_{r}$ and $w_{r}$ are distributed as i.i.d. $\mathcal{N}(0,1)$ vectors, normalized to satisfy $\left\|v_{r}\right\|=\left\|w_{r}\right\|=1$. This follows from the rotational invariance of the distribution of $e$ when $e_{i t}$ is i.i.d. normally distributed. Using this characterization of $v_{r}$ and $w_{r}$, one can formally show that Assumption EV(iii) holds; see Lemma A. 4 below. The conjecture in the random matrix theory literature is that the limiting distribution of the eigenvectors of a random covariance matrix is "distribution-free," that is, is independent of the particular distribution of $e_{i t}$ (see, e.g., Silverstein (1990), Bai (1999)). However, we are not aware of a formulation and corresponding proof of this conjecture that is sufficient for our purposes, which is one reason why we have to impose i.i.d. normality of $e_{i t}$.

(iii) Assumption EV(ii) imposes a condition on the eigenvalues $\rho_{r}$ of the random covariance matrix $M_{f^{0}} e^{\prime} M_{\lambda^{0}} e M_{f^{0}}$. Eigenvalues are studied more intensely than eigenvectors in the random matrix theory literature, and it is well known that the properly normalized empirical distribution of the eigenvalues (the so-called empirical spectral distribution) of an i.i.d. sample covariance matrix converges to the Marčenko-Pastur law (Marčenko and Pastur (1967)) for asymptotics, where $N$ and $T$ grow at the same rate. This means that the sum over the function of the eigenvalues $\rho_{s}$ in Assumption EV(ii) can be approximated by an integral over the Marčenko-Pastur limiting spectral distribution. To bound the asymptotic error of this approximation, one needs to know the convergence rate of the empirical spectral distribution to its limit law, which is an ongoing research subject in the literature, for example, Bai (1993), 
Bai, Miao, and Yao (2003), and Götze and Tikhomirov (2010). This literature usually considers either i.i.d. or i.i.d. normal distributions of $e_{i t}$.

(iv) For random covariance matrices from i.i.d. normal errors, it is known from Johnstone (2001) and Soshnikov (2002) that the properly normalized few largest eigenvalues converge to the Tracy-Widom law. ${ }^{45}$ This result can be used to verify Assumption EV(i) in the case of i.i.d. normal $e_{i t}$.

(v) Details on how to derive Theorem A.3 are given in Section S.4 of the Supplemental Material.

The following lemma provides the connection between Theorem A.3 and our main result, Theorem 3.1. The proof is given in the Supplemental Material.

LemmA A.4: Let Assumption LL hold, let $R^{0}=\operatorname{rank}\left(\lambda^{0}\right)=\operatorname{rank}\left(f^{0}\right)$, and consider a limit $N, T \rightarrow \infty$ with $N / T \rightarrow \kappa^{2}, 0<\kappa<\infty$. Then Assumptions SN, $\mathrm{EX}, \mathrm{DX}-1$, and $\mathrm{EV}$ are satisfied, and we have $C^{(1)}=\mathcal{O}_{P}(1)$.

\section{REFERENCES}

AHN, S. C., AND A. R. Horenstein (2013): "Eigenvalue Ratio Test for the Number of Factors," Econometrica, 81 (3), 1203-1227. [1555,1561,1562]

AHN, S. C., Y. H. LEE, AND P. SCHMIDT (2001): "GMM Estimation of Linear Panel Data Models With Time-Varying Individual Effects," Journal of Econometrics, 101 (2), 219-255. [1543,1544]

_ (2013): "Panel Data Models With Multiple Time-Varying Individual Effects," Journal of Econometrics, 174 (1), 1-14. [1543]

Allen, D. W. (1992): "Marriage and Divorce: Comment," American Economic Review, 82, 679-685. [1560]

ANDREws, D. W. K. (1999): "Estimation When a Parameter Is on a Boundary,” Econometrica, 67 (6), 1341-1384. [1556]

BAI, J. (2009a): "Panel Data Models With Interactive Fixed Effects," Econometrica, 77 (4), 1229-1279. [1543,1544,1547,1549-1551,1555,1557,1558,1563,1567,1571]

(2009b): "Supplement to 'Panel Data Models With Interactive Fixed Effects': Technical Details and Proofs," Econometrica Supplemental Material, 77, http://dx.doi.org/10.3982/ ECTA6135. [1544,1555]

_ (2013): "Likelihood Approach to Small T Dynamic Panel Models With Interactive Effects," Unpublished Manuscript. [1543]

BAI, J., AND S. NG (2002): "Determining the Number of Factors in Approximate Factor Models," Econometrica, 70 (1), 191-221. [1549,1555,1561,1562]

BAI, Z. (1993): "Convergence Rate of Expected Spectral Distributions of Large Random Matrices. Part II. Sample Covariance Matrices,” The Annals of Probability, 21 (2), 649-672. $[1560,1576]$

[1560 (1999): "Methodologies in Spectral Analysis of Large Dimensional Random Matrices, a Review," Statistica Sinica, 9, 611-677. [1559,1576]

BAI, Z., B. MiAO, AND J. YAO (2003): "Convergence Rates of Spectral Distributions of Large Sample Covariance Matrices," SIAM Journal on Matrix Analysis and Applications, 25 (1), 105-127. [1560,1577]

\footnotetext{
${ }^{45}$ To our knowledge, this result is not established for error distributions that are not normal. Soshnikov (2002) has a result under nonnormality but only for asymptotics with $N / T \rightarrow 1$.
} 
BAI, Z. D., J. W. Silverstein, AND Y. Q. YIN (1988): "A Note on the Largest Eigenvalue of a Large Dimensional Sample Covariance Matrix," Journal of Multivariate Analysis, 26 (2), 166-168. [1554]

Chudik, A., M. H. Pesaran, And E. Tosetti (2011): "Weak and Strong Cross-Section Dependence and Estimation of Large Panels," The Econometrics Journal, 14 (1), C45-C90. $[1545,1557]$

CoX, D. D., AND T. Y. KIM (1995): "Moment Bounds for Mixing Random Variables Useful in Nonparametric Function Estimation," Stochastic Processes and Their Applications, 56 (1), 151-158. [1571]

DHAENE, G., AND K. Jochmans (2015): "Split-Panel Jackknife Estimation of Fixed-Effect Models," Review of Economic Studies (forthcoming). [1553]

FriedBerG, L. (1998): "Did Unilateral Divorce Raise Divorce Rates? Evidence From Panel Data,” Technical Report, JSTOR. [1560,1561]

GEMAN, S. (1980): "A Limit Theorem for the Norm of Random Matrices," The Annals of Probability, 8 (2), 252-261. [1554]

GötZE, F., AND A. TIKHOMIRov (2010): "The Rate of Convergence of Spectra of Sample Covariance Matrices," Theory of Probability and Its Applications, 54, 129-140. [1560,1577]

GRAY, J. S. (1998): "Divorce-Law Changes, Household Bargaining, and Married Women's Labor Supply," American Economic Review, 88, 628-642. [1560]

HolTZ-EAKIN, D., W. NEWEY, AND H. S. RoSEN (1988): "Estimating Vector Autoregressions With Panel Data," Econometrica, 56 (6), 1371-1395. [1543,1550]

Johnstone, I. (2001): "On the Distribution of the Largest Eigenvalue in Principal Components Analysis," The Annals of Statistics, 29 (2), 295-327. [1560,1577]

KATO, T. (1980): Perturbation Theory for Linear Operators. Berlin: Springer-Verlag. [1556,1572]

KIEFER, N. (1980): "A Time Series-Cross Section Model With Fixed Effects With an Intertemporal Factor Structure," Unpublished Manuscript, Department of Economics, Cornell University. [1544]

KIM, D., AND T. OKA (2014): "Divorce Law Reforms and Divorce Rates in the USA: An Interactive Fixed-Effects Approach,” Journal of Applied Econometrics, 29, 231-245. [1546,1560,1561, 1563]

Latala, R. (2005): "Some Estimates of Norms of Random Matrices," Proceedings of the American Mathematical Society, 133, 1273-1282. [1554,1570]

LU, X., AND L. SU (2013): "Shrinkage Estimation of Dynamic Panel Data Models With Interactive Fixed Effects,” Working Paper, Hong Kong University of Science \& Technology. [1543]

MARČENKO, V., AND L. PASTUR (1967): "Distribution of Eigenvalues for Some Sets of Random Matrices," Sbornik: Mathematics, 1 (4), 457-483. [1560,1576]

MoOn, H., AND M. WeIDNER (2014): "Dynamic Linear Panel Regression Models With Interactive Fixed Effects," Working Paper, CeMMAP. [1543,1544,1550,1551,1554,1557,1563,1570]

(2015): "Supplement to 'Linear Regression for Panel With Unknown Number of Factors as Interactive Fixed Effects'," Econometrica Supplemental Material, 83, http://dx.doi.org/ 10.3982/ECTA9382. [1546]

Moon, H., M. Shum, AND M. WeIDner (2014): "Interactive Fixed Effects in the blp Random Coefficients Demand Model," Working Paper, CeMMAP. [1554]

Nickell, S. (1981): "Biases in Dynamic Models With Fixed Effects," Econometrica, 49 (6), 1417-1426. [1551]

ONATSKI, A. (2010): "Determining the Number of Factors From Empirical Distribution of Eigenvalues," Review of Economics and Statistics, 92 (4), 1004-1016. [1545,1555,1557,1561,1562]

(2012): "Asymptotics of the Principal Components Estimator of Large Factor Models With Weakly Influential Factors," Journal of Econometrics, 168 (2), 244-258. [1545,1557]

(2013): "Asymptotic Analysis of the Squared Estimation Error in Misspecified Factor Models," Unpublished Manuscript. [1570]

Pesaran, M. H. (2006): "Estimation and Inference in Large Heterogeneous Panels With a Multifactor Error Structure," Econometrica, 74 (4), 967-1012. [1543,1544,1550] 
Peters, H. E. (1986): "Marriage and Divorce: Informational Constraints and Private Contracting," American Economic Review, 76 (3), 437-454. [1560]

(1992): "Marriage and Divorce: Reply," American Economic Review, 82, 686-693. [1560]

Silverstein, J. W. (1989): "On the Eigenvectors of Large Dimensional Sample Covariance Matrices," Journal of Multivariate Analysis, 30 (1), 1-16. [1554]

_ (1990): "Weak Convergence of Random Functions Defined by the Eigenvectors of Sample Covariance Matrices,” The Annals of Probability, 18 (3), 1174-1194. [1559,1576]

SoshniKov, A. (2002): "A Note on Universality of the Distribution of the Largest Eigenvalues in Certain Sample Covariance Matrices," Journal of Statistical Physics, 108 (5), 1033-1056. [1560, 1577]

StOcK, J. H., AND M. W. WATSON (2002): "Forecasting Using Principal Components From a Large Number of Predictors," Journal of the American Statistical Association, 97, 1167-1179. [1549]

VERSHYNIN, R. (2012): "Introduction to the Non-Asymptotic Analysis of Random Matrices," in Compressed Sensing. Cambridge: Cambridge University Press, 210-268. [1570]

WolfERS, J. (2006): "Did Unilateral Divorce Laws Raise Divorce Rates? A Reconciliation and New Results," American Economic Review, 96, 1802-1820. [1560,1561,1563]

YIN, Y. Q., Z. D. BAI, AND P. KRISHNAIAH (1988): "On the Limit of the Largest Eigenvalue of the Large-Dimensional Sample Covariance Matrix," Probability Theory and Related Fields, 78, 509-521. [1554]

ZAFFARONI, P. (2009): “Generalized Least Squares Estimation of Panel With Common Shocks," Unpublished Manuscript. [1543]

Dept. of Economics and USC Dornsife INET, University of Southern California, Los Angeles, CA 90089-0253, U.S.A. and Dept. of Economics, Yonsei University, Seoul, Korea; moonr@usc.edu

and

Dept. of Economics, University College London, Gower Street, London, WC1E 6BT, U.K. and CeMMaP; m.weidner@ucl.ac.uk.

Manuscript received June, 2010; final revision received December, 2014. 